ARTICLE

\title{
A robust and tunable halogen bond organocatalyzed 2-deoxyglycosylation involving quantum tunneling
}

\author{
Chunfa Xu (iD) ${ }^{1,2}$, V. U. Bhaskara Rao ${ }^{1,2}$, Julia Weigen ${ }^{1,2} \&$ Charles C. J. Loh (1) ${ }^{1,2}$
}

The development of noncovalent halogen bonding $(X B)$ catalysis is rapidly gaining traction, as isolated reports documented better performance than the well-established hydrogen bonding thiourea catalysis. However, convincing cases allowing $X B$ activation to be competitive in challenging bond formations are lacking. Herein, we report a robust $X B$ catalyzed 2-deoxyglycosylation, featuring a biomimetic reaction network indicative of dynamic $X B$ activation. Benchmarking studies uncovered an improved substrate tolerance compared to thioureacatalyzed protocols. Kinetic investigations reveal an autoinductive sigmoidal kinetic profile, supporting an in situ amplification of a XB dependent active catalytic species. Kinetic isotopic effect measurements further support quantum tunneling in the rate determining step. Furthermore, we demonstrate XB catalysis tunability via a halogen swapping strategy, facilitating 2-deoxyribosylations of D-ribals. This protocol showcases the clear emergence of XB catalysis as a versatile activation mode in noncovalent organocatalysis, and as an important addition to the catalytic toolbox of chemical glycosylations.

\footnotetext{
${ }^{1}$ Abteilung Chemische Biologie, Max Planck Institut für Molekulare Physiologie, Otto-Hahn-Straße 11, 44227 Dortmund, Germany. ${ }^{2}$ Fakültät für Chemie und

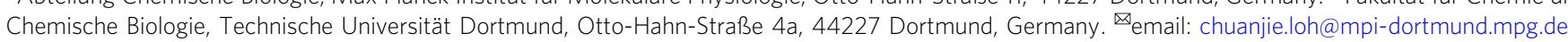


$\mathrm{N}$ oncovalent catalysis constitutes one of the major pillars of organocatalysis $^{1-4}$, by capitalizing intermolecular interactions, such as hydrogen bonds (HB) to activate specific functional groups under mild conditions. Indisputably, $\mathrm{HB}$ catalysis ${ }^{5,6}$ dominates the majority of noncovalent organocatalyzed protocols, which is largely spearheaded by thiourea catalysis $^{7-14}$. Furthermore, thiourea organocatalysis is widely dubbed as biomimetic ${ }^{2}$, as it derives inspiration from how enzymes capitalize noncovalent interactions to catalyze biochemical reactions ${ }^{1-4}$. Hence, biomimetic thiourea catalysis is well recognized as a powerful synthetic tool spanning from asymmetric catalysis to natural product synthesis ${ }^{5-14}$.

In contrast, while the utility of the more directional halogen bond $(\mathrm{XB})$ is increasingly establishing its prominence in noncovalent organocatalysis ${ }^{15-21}$, mechanistic understanding and utility of unique $\mathrm{XB}$ activation modes is highly limited. While nature also exploits XBs in catalytic processes ${ }^{22}$, for instance in thyroid hormones, the development of biomimetic XB catalytic strategies in constructing biologically important molecules is surprisingly underexplored in the literature.

In recent years, despite the development of excellent proof-ofconcept XB-catalyzed reactions by multiple research groups ${ }^{23-42}$, protocols whereby $\mathrm{XB}$ catalysis shows clear catalytic advantages in over conventional thiourea catalysis is lacking ${ }^{21}$. Moreover, a conceivable configurational advantage pertaining to $\mathrm{XB}$ catalysis would involve the ease of $\sigma$-hole tunability via halogen swapping to accommodate challenging substrates-an advantage not well adoptable in thiourea catalysis due to strict requirements of hydrogen atoms for dual $\mathrm{HB}$ activation. This property is however not yet successfully exploited. In addition, the majority of currently reported $\mathrm{XB}$ catalytic strategies are limited to monofunctional group activation, such as solely on carbonyls, imines, quinolines, iodonium ylides, or halides ${ }^{15-21}$. Biomimetic complex kinetic behavior involving in situ dynamic XB activation on multiple reaction species offers great promise in unraveling unknown $\mathrm{XB}$ mechanisms and reactivity, but little is currently known.

In an elegant seminal benchmarking case, Huber et al. demonstrated a XB-catalyzed Diels-Alder reactions between cyclopentadiene and methyl vinyl ketone (Fig. 1a) through XBketone activation ${ }^{27}$. A comparison of the kinetic profiles between $\mathrm{XB}$ and thiourea catalysts illuminated superior XB catalytic performance. An extension of the same activation concept was exemplified by Toy et al., wherein they demonstrated substantially improved effectiveness of a bidentate XB catalyst over thiourea catalysis in a bis-Friedel-Crafts-type reaction of indoles on ketones ${ }^{41}$.

Furthermore, Takemoto and coworkers showcased in 2017 a powerful cross-enolate coupling reaction catalyzed by a monodentate XB catalyst, which furnished superior yield over thiourea catalysis (Fig. 1b) ${ }^{32}$. Recent DFT calculations by Wong et al. offer theoretical basis into the potential competitiveness of XB catalysis with respect to thiourea catalysis ${ }^{43}$. Lately, Yeung and coworkers also reported that $\mathrm{XB}$ catalysis enables addition of silylated $C$ nucleophiles to $\mathrm{N}$-acyliminium ions (Fig. 1c), which was unachievable using thiourea catalysis ${ }^{35}$. Our group also demonstrated in 2019 that a multistage XB catalysis gave broadly superior anomeric selectivity in strain-release glycosylations ${ }^{44}$, (Fig. 1d) superceding that of our earlier reported thiourea-catalyzed protocol ${ }^{45}$. These very limited prior examples provide pressing impetus for more experimental proof and deeper mechanistic understanding of $\mathrm{XB}$ catalysis, as a high-performance noncovalent catalytic tool in challenging reactions.

Specifically, we aim to harness and unravel noncovalent mechanisms unique to $\mathrm{XB}$ organocatalysis, so that enabling methodologies, which facilitates the preferential construction of challenging bonds over thiourea catalysis in biologically relevant molecules can be developed. In line with this aim, we have identified the biologically relevant 2-deoxyglycosylation as an ideal reaction model for the discovery for unknown XB catalytic mechanisms ${ }^{46-59}$. The unique poly-oxygenated nature of glycosyl substrates and products provides numerous $\mathrm{XB}$ acceptor moieties for the in situ catalytic establishment of dynamic noncovalent activations. Moreover, the synthetic importance of 2deoxyglycosides as a privileged and biologically useful compound class is well exemplified by the continual intense interest by many different research groups ${ }^{46-59}$, due to its prevalence in glycosidic natural products, such as digitoxin and saccharomicin B (ref. ${ }^{46}$ ).

On the organocatalytic front ${ }^{60-64}$, seminal studies by Galan and McGarrigle et al. demonstrated the utility of thiourea catalysis in accessing 2-deoxyglycosides via mild organocatalytic conditions ${ }^{47,56}$. However, significant limitations still exist in such protocols. For instance, the sole application of thiourea catalysis limits the donor scope to galactals ${ }^{47}$, and expansion to challenging glycosyl donors, such as glucals and rhamnals required harsher conditions, i.e., the usage of either a conventional strong Brønsted acid $^{48}$, or a judiciously matched combination of thiourea and an appropriate enantiomer of a strong chiral Brønsted acid ${ }^{49}$. A caveat of directly employing strong Brønsted acid activators lies in the product decomposition due to greatly enhanced hydrolytic cleavage, of up to 2000-fold elevated acid labilities of 2-deoxyglycosides (Supplementary Note 14) ${ }^{65}$, which necessitates rigorous water exclusion procedures, such as extended pre-vacuum suctioning of substrates ${ }^{47-49,56}$. As the critical mechanistic influence of activators and catalysts in glycosylation pathways is well recognized but largely understudied ${ }^{66}$, the recent realization of unconventional XB catalytic mechanisms offers huge potential to expand the glycosylation activator toolbox ${ }^{15-21}$ and to accommodate improved substrate scopes, while retaining mild conditions required for broad substrate utility and circumventing decomposition.

Furthermore, the applicability of $\mathrm{XB}$ activation in advancing chemical glycosylations is still in its infancy. Apart from our above mentioned XB-catalyzed strain-release glycosylation ${ }^{44}$, Huber and Codée et al. demonstrated a seminal stoichiometric proof-of-principle XB activation in Koenigs-Knorr glycosylation $^{67}$, and recently Takemoto et al. described a powerful XB-cocatalytic role to elevate the Brønsted acidity in thiourea for $\mathrm{N}$ glycofunctionalizations and $N$-glycosylations ${ }^{68,69}$.

We herein report a remarkable XB-catalyzed 2-deoxyglycosylation of glycals 1 (Fig. 1e), featuring robustness, mildness, substrate broadness, and mechanistic complexity via a switchable intricate reaction network. Noncovalent catalytic benchmarking studies are conducted between our protocol and widely established thiourea-catalyzed protocols 47,56 , and our protocol furnishes overall superior donor and acceptor substrate generality, noteworthily in silylated galactals, glucals, rhamnals, and pentose-derived donors. We introduce a halogen swapping strategy for challenging D-ribal substrates, and this was found to be effective in tolerating challenging 2-deoxypyranoribosylations. In situ NMR monitoring reveal a sigmoidal kinetic profile characteristic of autoinduction, suggesting an amplificative formation of an in situ catalyst. Kinetic isotopic effect (KIE) experiments suggest that quantum tunneling is operative in the proton transfer rate-determining step of the mechanism.

\section{Results}

Establishment of an XB-catalyzed 2-deoxyglycosylation. We initiated our investigation on a model glycosylation by selecting the D-glucal substrates $\mathbf{1 a - 1} \mathbf{b}$ as our glycosyl donor, and $\mathbf{2 a}$ as the 


\section{Limited prior reports of competitiveness of XB over thiourea catalysis}

a Huber et al. (2014)

Diels-Alder reaction

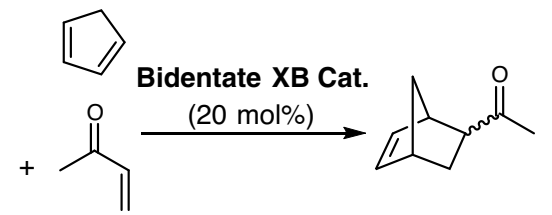

$\checkmark$ Superior temporal kinetic performance over thiourea

C Yeung et al. (2019)

Addition to $\mathrm{N}$-acyliminium ions

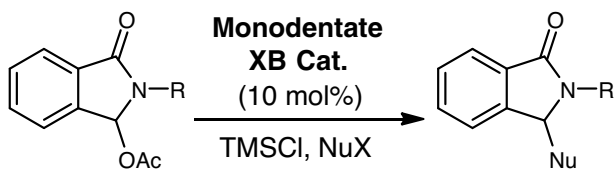

b Takemoto et al. (2017)

Cross-enolate coupling

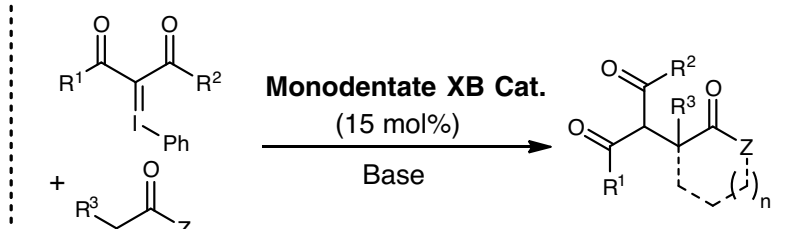

$+{ }^{+}$

( $\left(\begin{array}{l}z \\ j\end{array}\right)_{n} \checkmark$ Superior yield over thiourea

d Loh et al. (2019)

Multistage XB catalyzed strain-release glycosylation

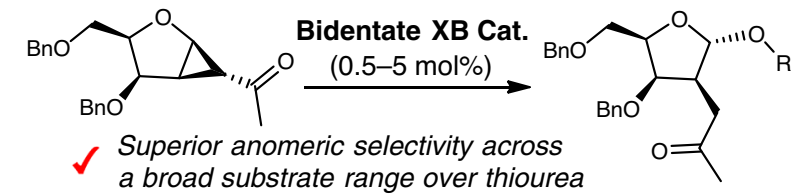
a broad substrate range over thiourea

$\checkmark$ Superior XB reactivity over thiourea

$\checkmark$ Mechanistic distinctiveness over thiourea

e Current work:<smiles>O=POC1C=C[Te]C1</smiles>

$+\mathrm{ROH}$

2<smiles></smiles>

(3 $\mathrm{mol} \%)$

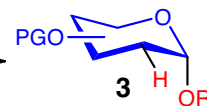

Overall broader substrate tolerances over thioureas

$\checkmark$ Mechanistic distinctiveness of XB catalysis over thiourea

$\checkmark$ Quantum tunneling in the rate determining step

ON/OFF switchable kinetic modulated by XBs

$X B$ catalyst tunability via halogen swapping

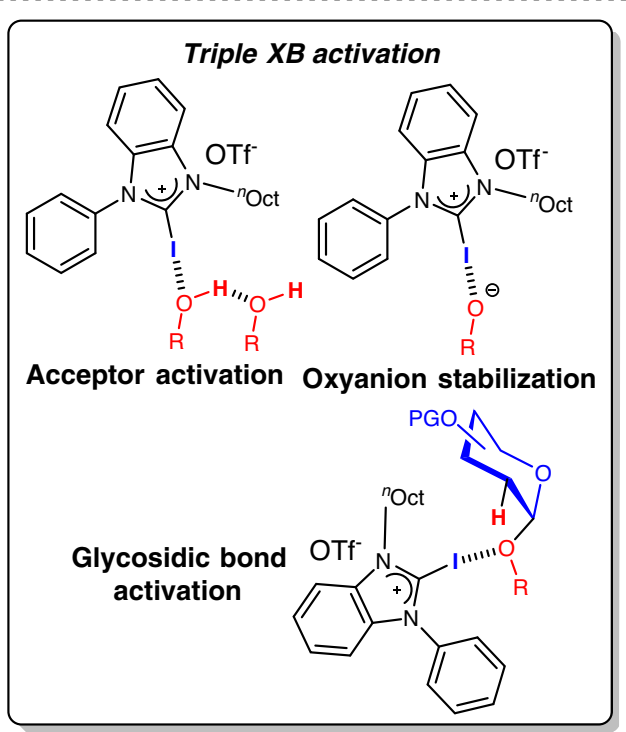

Fig. 1 Prior reports in enhanced XB over thiourea based HB catalysis. a XB-catalyzed Diels-Alder reaction. $\mathbf{b}$ XB-catalyzed cross-enolate coupling reaction. c XB-catalyzed Friedel-Crafts-type bis-indolylation. d XB-catalyzed strain-release glycosylation. e Current work.

acceptor. Utilizing the benzylated D-glucal 1a (Fig. 2, entry 1-3) was deleterious in our reaction because of substantial formation of Ferrier side products. Encouragingly, when silylated D-glucal $1 \mathbf{b}$ (Fig. 2, entry 4) was employed ${ }^{48}$, we observed a yield elevation of $3 \mathrm{a}$ to $92 \%$ with excellent anomeric selectivity. A screen of various $\mathrm{XB}$ catalysts $\mathbf{A}-\mathbf{D}$ revealed that $\mathbf{A}$ is optimal for our reaction ${ }^{32}$. We eventually arrived at our optimized protocol (Fig. 2, entry 18), whereby only $3 \mathrm{~mol} \%$ of catalyst $\mathbf{A}$ is required for the glycosylation, furnishing $\mathbf{3 a}$ with $89 \%$ yield and excellent anomeric selectivity (>20:1).

Control experiments for confirming XB catalysis. To better understand the possible interference effects due to trace acidic HI generation, a series of control experiments were conducted ${ }^{21}$. While the non-iodinated control catalyst $\mathbf{E}$ gave good yields of $\mathbf{3 a}$ (Fig. 2, entry 8), the benzimidazolium hydrogen is known to be acidic and could function as either a nonclassical HB or a Brønsted acid catalyst (Supplementary Note 15$)^{70,71}$, and does not constitute exclusionary proof of $\mathrm{XB}$ catalysis by $\mathrm{A}$. To better investigate the criticality of XB catalyst influences on our system, TBAB and TBAI are added into the reaction to inhibit the $\mathrm{XB}$ activation mechanism by competition for the $\sigma$-hole site, due to the extremely high affinity of halide ions to the XB catalyst (Fig. 2, entries 9 and 10) 25,29 . Both halide competition experiments gave negligible yield $(<5 \%)$ of $\mathbf{3 a}$, further augmenting the presence of $\mathrm{XB}$ catalysis.

As parallel controls to ascertain $\mathrm{TBAB}$ poisoning effects on conventional Brønsted acids without XB influence, the glycosylation was carried out twice in catalytic amounts of $\mathrm{HCl}(5 \mathrm{~mol} \%)$, a surrogate for $\mathrm{HI}$, in the absence and presence of the competition reagent $\mathrm{TBAB}$ (Fig. 2, entries 11 and 12). The similarly good yields of $3 \mathbf{a}$ in both control experiments ( $80-86 \%$ NMR yield) signify that TBAB competition has no effect on fortuitous acid catalysis, further solidifying the cruciality of $\mathrm{XB}$ catalysis in our protocol. In control experiments simply reacting $\mathbf{A}$ with isopropanol, the absence of $\mathrm{O}$-acceptor substituted benzimidazolium side products in LC-MS and ${ }^{1} \mathrm{H}$ NMR analysis of the reaction mixture further supports the absence of trace acid catalysis (Supplementary Figs. S5-S9 and Supplementary Note 16).

Furthermore, the addition of catalytic amounts of organic base $\mathbf{F}$ (Fig. 2, entries 13 and 14), or inorganic base $\mathrm{K}_{2} \mathrm{CO}_{3}$ (Fig. 2, 


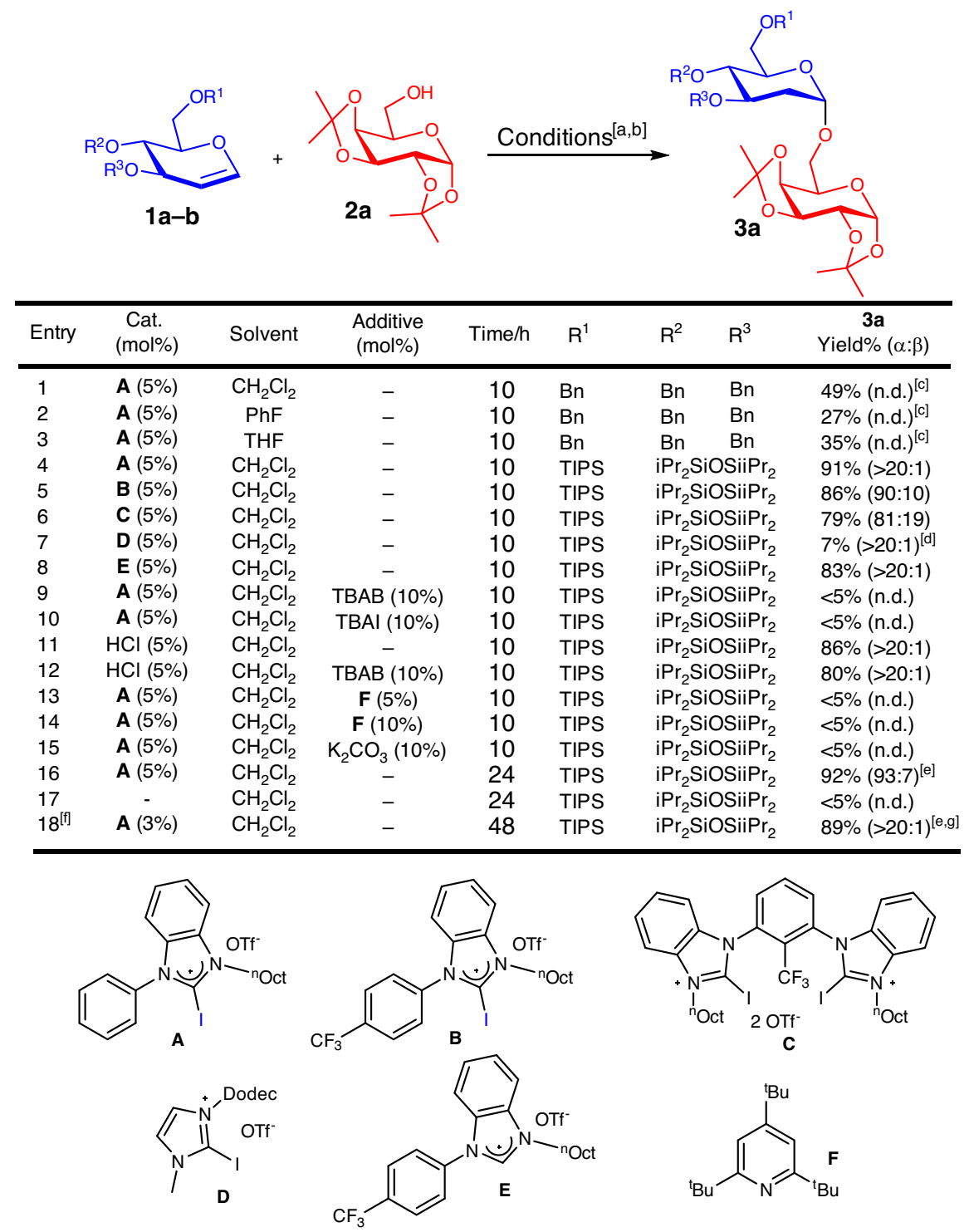

Fig. 2 Optimization of XB-catalyzed 2-deoxyglycosylation. [a] 2a $(0.1 \mathrm{mmol})$, 1a-b $(0.15 \mathrm{mmol})$, catalyst, $50^{\circ} \mathrm{C}$, solvent $(0.2 \mathrm{M})$, time, argon. [b] Yield and $\alpha: \beta$ ratio were determined by crude ${ }^{1} \mathrm{H}$ NMR spectra analysis using 1,3,5-trimethoxybenzene as an internal standard. [c] 40-50\% NMR yields of Ferrier rearrangement side-products detected. [d] $92 \% \mathbf{1 b}$ remaining. [e] 2a $(0.2 \mathrm{mmol}), \mathbf{1 b}(0.30 \mathrm{mmol})$, catalyst, $50^{\circ} \mathrm{C}, \mathrm{CH}_{2} \mathrm{Cl}_{2}(0.2 \mathrm{M})$, time, argon. $[\mathrm{f}]$ Conducted at $40^{\circ} \mathrm{C}$. [g] Isolated yields. n.d. not determined, iPr isopropyl, TBAB tetrabutylammonium bromide, TBAI tetrabutylammonium iodide.

entry 15$)$ terminated the glycosylation $(<5 \%$ yield $)$. This observation is in contrast with our previously reported XBcatalyzed strain-release glycosylation ${ }^{44}$, whereby the reaction still proceeded with relatively good yields in the presence of base. A control experiment in the absence of catalyst $\mathbf{A}$ did not result in reaction (Fig. 2, entry 17). Evaluation of these control experiments revealed a strong dependency on $\mathrm{XB}$ catalysis in this protocol. The presence of base deactivation does not necessarily confirm the presence of trace acidity, if $\mathrm{XB}$ activation is crucial in initiating a proton transfer step, and the base serves as quencher of the proton transfer. These observations point us toward a possible complex interplay of mechanisms through dynamic XB interactions in our methodology.

Substrate scope. With an optimized protocol in hand, we proceeded to establish the substrate scope of the protocol. We determined that this protocol tolerates a wide range of hexosebased donors $\mathbf{1 b} \mathbf{b} \mathbf{1 h}$, furnishing glycosides $\mathbf{3 a}$-at with good to excellent yields and generally excellent anomeric selectivity
(Fig. 3). For the hexoses-based donors 1, natural and nonnatural D- and L-glucal substrates, D- and L-galactal, and L-rhamnalbased substrates are very well tolerated in this methodology. Generally, a range of different $O$-acceptors bearing the free hydroxyl moiety at multiple positions are very well tolerated to yield the target glycosides 3 with excellent anomeric selectivity. This include the protected monosaccharides acceptors to generate $\mathbf{3 a - 3 j}, \mathbf{3 s - 3 a c}$, and 3an-3ar. Steroidal acceptors such as cholesterol, testosterone, and methyltestosterone are also well accommodated within the different hexoses donors (3k-3m, 3ad-3ag, and 3as). Interestingly, protected amino acid residues, including L-serine, L-threonine, and L-tyrosine work well in our protocol to generate glycopeptide-type derivatives (3n-3q, 3ah-3aj, and 3at). Simple primary and secondary alcohols, such as propargyl alcohol and isopropanol also gave the target glycosides with generally good to excellent yields, and very good to excellent selectivity (3r and 3ak-3al).

Furthermore, we sought to investigate whether our protocol is robust enough to accommodate less facile pentoses-based donors 
Hexoses donor scope

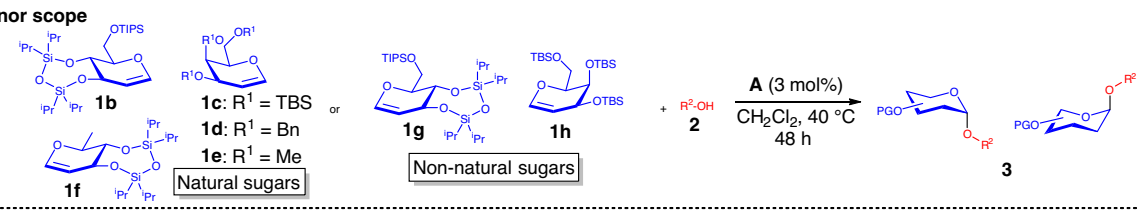
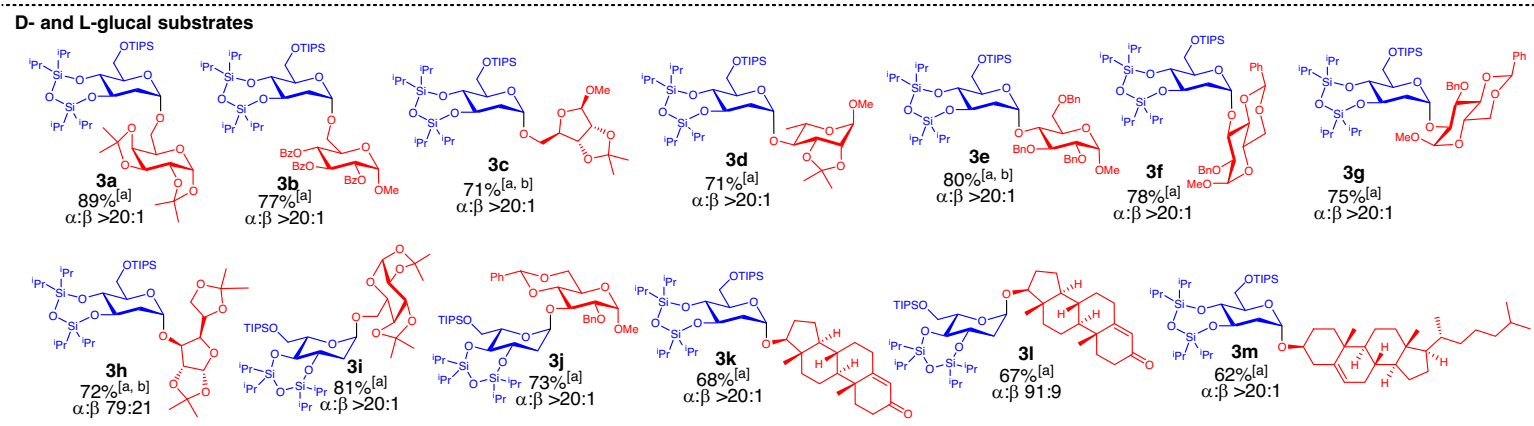

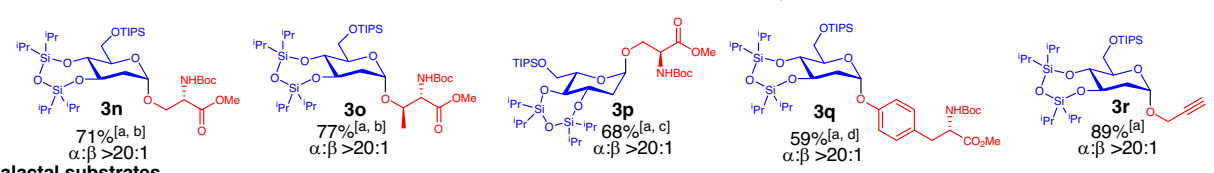

D- and L-galactal substrates
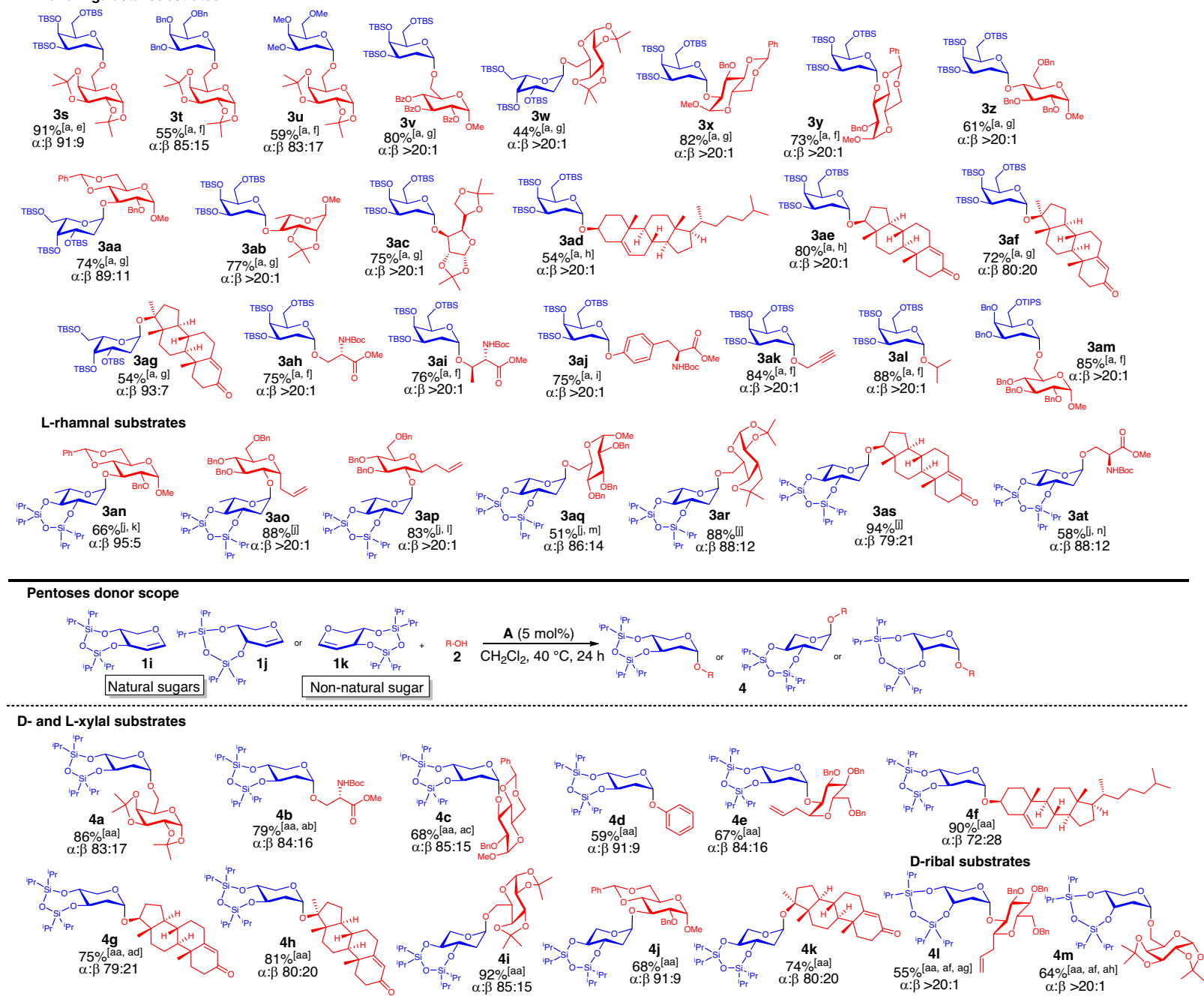

Fig. 3 Substrate scope of the XB-catalyzed glycosylation. Hexoses donor scope: $[a] \mathbf{2}(0.2 \mathrm{mmol})$, $\mathbf{1 b}-\mathbf{1 h}(0.3 \mathrm{mmol})$ and $3 \mathrm{~mol} \%$ catalyst $\mathbf{A}$, in $\mathrm{CH}_{2} \mathrm{Cl}_{2}$ $(1 \mathrm{~mL})$, argon, $40^{\circ} \mathrm{C}, 48 \mathrm{~h} ; \alpha: \beta$ ratio was determined by crude ${ }^{1} \mathrm{H}$ NMR analysis. [b] 5 mol\% catalyst $\mathbf{A}, 50^{\circ} \mathrm{C}$. [c] 5 mol\% catalyst $\mathbf{A}, 50{ }^{\circ} \mathrm{C}, 72 \mathrm{~h}$. [d] $5 \mathrm{~mol}$ $\%$ catalyst A, $50{ }^{\circ} \mathrm{C}, 108 \mathrm{~h}$. [e] RT, $24 \mathrm{~h}$. [f] $30^{\circ} \mathrm{C}$. [g] $30^{\circ} \mathrm{C}, 72 \mathrm{~h}$. [h] $30^{\circ} \mathrm{C}, 24 \mathrm{~h}$. [i] $10 \mathrm{~mol} \%$ catalyst $\mathbf{A}, 40^{\circ} \mathrm{C}, 96 \mathrm{~h}$. [j] $2(0.2 \mathrm{mmol}), 1 \mathbf{f}(0.4 \mathrm{mmol})$ and $5 \mathrm{~mol} \%$ catalyst $\mathbf{A}$, in $\mathrm{CH}_{2} \mathrm{Cl}_{2}(1 \mathrm{~mL})$, argon, $30^{\circ} \mathrm{C}, 48 \mathrm{~h}$. [k] $108 \mathrm{~h}$. [l] $40^{\circ} \mathrm{C}$. [m] $96 \mathrm{~h}$. [n] $50{ }^{\circ} \mathrm{C}, 72 \mathrm{~h}$. Pentoses donor scope: [aa] $\mathbf{2}$ (0.2 mmol), 1i-k $(0.3 \mathrm{mmol})$ and $5 \mathrm{~mol} \%$ catalyst $\mathbf{A}$, in $\mathrm{CH}_{2} \mathrm{Cl}_{2}(1 \mathrm{~mL})$, argon, $40^{\circ} \mathrm{C}, 24 \mathrm{~h} ; \alpha$ : $\beta$ ratio was determined by crude ${ }^{1} \mathrm{H} \mathrm{NMR}$ analysis. [ab] $96 \mathrm{~h}$. [ac] $30{ }^{\circ} \mathrm{C}, 48 \mathrm{~h}$. [ad] $48 \mathrm{~h}$. [ae] $3 \mathrm{~mol} \%$ catalyst $\mathbf{A}, 30^{\circ} \mathrm{C}, 48 \mathrm{~h}$. [af] $\mathbf{1 j}(0.4 \mathrm{mmol})$. [ag] $3 \mathrm{~mol} \%$ catalyst $\mathbf{A}, 35^{\circ} \mathrm{C}, 48 \mathrm{~h}$. [ah] $3 \mathrm{~mol} \%$ catalyst $\mathbf{A}, 30^{\circ} \mathrm{C}$. RT $=$ room temperature $23^{\circ} \mathrm{C}, \mathrm{PG}=$ Protecting Group. 
1i-1k, which are challenging in thiourea catalysis ${ }^{47,56}$. Gratifyingly, natural $(\mathbf{1 i}, \mathbf{1} \mathbf{j})$ and nonnatural pentose-based donors $(\mathbf{1 k})$ work generally well in our XB catalysis protocol, to generate the target glycosides $(\mathbf{4 a - 4 m})$ with good to excellent yields and good anomeric selectivity. In the case of the D-xylal substrate 1i (Fig. 3), while there is a clear anomeric preference toward the $a-$ anomer, a diminishment of anomeric selectivity was observed compared to the hexoses, probably due to absence of steric hindrance on the $\beta$-face from the $\mathrm{C} 5$ substituent. The nonnatural $\mathrm{L}$-xylal was tolerated in the XB catalytic protocol $(\mathbf{4 i}-\mathbf{4 k})$, furnishing anomeric selectivity in the similar range as the natural D-congeners.

We also investigated the use of D-ribal $\mathbf{1 j}$ to access the 2deoxyribose scaffold in $\mathbf{4 1 - 4 m}$. Glycosidic linkages to 2deoxypyranoribose can be successfully constructed using glycosyl acceptors bearing free primary or secondary alcohol groups, with generally moderate to good yields and excellent anomeric selectivity (Fig. 3).

The overall substrate scope indicated broader donor and acceptor tolerance over established thiourea catalysis, particularly in accessing 2-deoxyglycosides from challenging glucals, rhamnals, and pentose-derived donors. It must be mentioned, however, that thiourea catalysis offers better tolerance on $\mathrm{D}$-galactal donors with ether protecting groups, while XB catalysis is superior when challenging secondary and phenolic acceptors are used on silylated D-galactal donors. Data on benchmarking studies comparing these substrates and protecting group influences against thiourea catalysis will be explored in a later section.

To further ascertain the presence of $\mathrm{XB}$ activation on a model glycosyl acceptor 2al, a ${ }^{13} \mathrm{C}$ NMR titration with the addition of increasing quantities of isopropanol $\mathbf{2 a l}$ to $\mathbf{A}$ was conducted (Fig. 4a). A downfield shift of the C-I carbon resonance in $\mathbf{A}$ of $\sim 1.9$ p.p.m. was observed. The direction of the NMR shift is in accordance with known literature reports of catalytic XB activation $^{27,29,44,68}$. Fitting of the host-guest titration to a $1: 1$ isotherm curve yielded an excellent fit with a $K_{\mathrm{a}}$ value of $0.45 \mathrm{M}^{-1}$ (Fig. 4b, Supplementary Fig. S2 and Supplementary Table S2). The TBAB and TBAI competition experiments were corroborated by ${ }^{13} \mathrm{C}$ NMR resonance shifts of the C-I carbon of catalyst $\mathbf{A}$ when an equimolar amount of either TBAB or TBAI was added (Supplementary Figs. S24 and S30) ${ }^{25}$. In the case of a 1:1 molar ratio of A:TBAB, a downfield shift of 15.45 p.p.m. of the ${ }^{13} \mathrm{C}$ resonance was observed. When a solution of a $1: 1$ molar ratio of A:TBAI was measured, an analogous downfield ${ }^{13} \mathrm{C}$ resonance shift of 14.34 p.p.m. was observed. The distinctively larger downfield shifts arising from interaction with TBAB and TBAI, compared to the 1:1 adduct formed between catalyst $\mathbf{A}$ and isopropanol (Fig. 4a and Supplementary Table S2) further support that halides, such as bromide and iodide bind much tighter to catalyst $\mathbf{A}$ than isopropanol ${ }^{25}$. Isothermal titration calorimetry (ITC) experiments between TBAB or TBAI with A yielded $K_{\mathrm{a}}$ values of 7050 and $2210 \mathrm{M}^{-1}$, respectively (Supplementary Figs. S3 and S4), confirming that halide ion binding to $\mathbf{A}$ is of significant orders of magnitude higher than an isopropanol to A interaction, reaffirming the value of halide competition experiments (Fig. 2) in ascertaining XB influences by poisoning the catalyst. A ${ }^{13} \mathrm{C}$ NMR experiment obtained by mixing a $1: 1$ molar ratio of donor $\mathbf{1 c}$ with catalyst $\mathbf{A}$ gave an almost negligible ${ }^{13} \mathrm{C}$ NMR shift of the C-I resonance (Supplementary Fig. S36). This supports the hypothesis that noncovalent interactions between the glycosyl donor and $\mathbf{A}$ are insignificant in the reaction mechanism ${ }^{38}$.

Mechanistic investigation by deuterated experiments. For a deeper insight into the mechanism, deuterated experiments
(Fig. 5 and Supplementary Fig. S44) were conducted. In a first control experiment (Fig. 5, Equation 1), the deuterated 2propanol-OD 5 was instead used as the acceptor in the XBcatalyzed glycosylation. We observed the formation of both the cis-addition product 6 and the trans-addition product 7 in a ratio of $9.5: 1(6: 7)$.

In a second confirmatory experiment, a galactal donor 8 with a deuterium label on $\mathrm{C} 2$ was synthesized and subjected to the standard XB catalytic conditions with isopropanol (Fig. 5, Equation 2). In this case, we detected the formation of both the cis-addition product 7 and the trans-addition product $\mathbf{6}$, in a product ratio of 13.5:1 (7:6). The scrambling of the deuterium labels on C2 in both experiments and the appearance of cis- and trans-addition products, suggests that the reaction proceeds through a step-wise reaction, indicating that protonation of the $\mathrm{C} 2$ of the glycal from the acceptor $-\mathrm{OH}$ proton constitute the first step of the mechanism.

Intermolecular competition experiments using equimolar amounts of isopropanol and deuterated 2-propanol-OD (Fig. 5, Equations 3-4, and Supplementary Figs. S39 and S40) were conducted to determine the presence of primary KIEs. We first conducted two experiments, each terminated at high and low conversions, respectively. In the former, a KIE value of 5.7 is obtained and in the latter, a KIE value of 13.3 is obtained. We postulate that this difference in primary KIE attained under different conversions might be attributed to a mechanistic shift in the overall mechanism as time proceeded, i.e., the initial significance of tunneling in the proton transfer rate-determining step is less pronounced, when a more predominant dynamic acceptor exchange cycle sets in the later part of the reaction (see mechanistic explanation in the later section of the manuscript), which can involve further proton transfer elementary steps. This relatively larger KIE values beyond the semiclassical limit $(>9)$ supports the involvement of quantum tunneling in the ratedetermining step ${ }^{72-78}$, where the conversion from reactants to products tunnels directly through a kinetic barrier without traversing an energetic maxima.

While such quantum tunneling phenomena were previously known in proton transfer reactions ${ }^{72-78}$, as well as in enzymatic catalysis $^{77,79,80}$, this is hitherto not well understood in the context of noncovalent organocatalysis. A further primary KIE value was determined using a nonpolar solvent mixture of $10: 1 \mathrm{PhCH}_{3}$ : $\mathrm{CH}_{2} \mathrm{Cl}_{2}$, and a substantial elevation of primary KIE to 23.4 was observed (Fig. 5, Equation 5 and Supplementary Fig. S43). This solvent dependence is in line with previously reported proton transfer reactions involving quantum tunneling ${ }^{81,82}$, as polar solvents increase the effective mass of the proton via coupling of solvent dipoles, which reduces tunneling and hence decreases the measured KIE.

Furthermore, competition experiments to determine secondary KIE were carried out (Fig. 5, Equations 6-7, and Supplementary Figs. S45 and S46). When equimolar amounts of isopropanol and 9 were used, a secondary KIE value of 1.16 was obtained. In addition, when galactals $\mathbf{1 c}$ and $\mathbf{8}$ were employed in an analogous experiment, a secondary KIE of 0.994 was obtained. The large primary KIEs and the close to unity values attained for secondary KIE experiments strongly support the possibility of $\mathrm{OH}$ bond breaking as the rate-determining step.

Mechanistic experiments via in situ NMR spectroscopy. We then undertook an in situ NMR monitoring experiment (Fig. 6) under standard conditions. Surprisingly, the experiment displayed a counter-intuitive sigmoidal kinetic profile ${ }^{83-85}$, differing from saturation kinetics observed our previously reported XBcatalyzed strain-release glycosylation ${ }^{44}$. Superimposition of the 
a
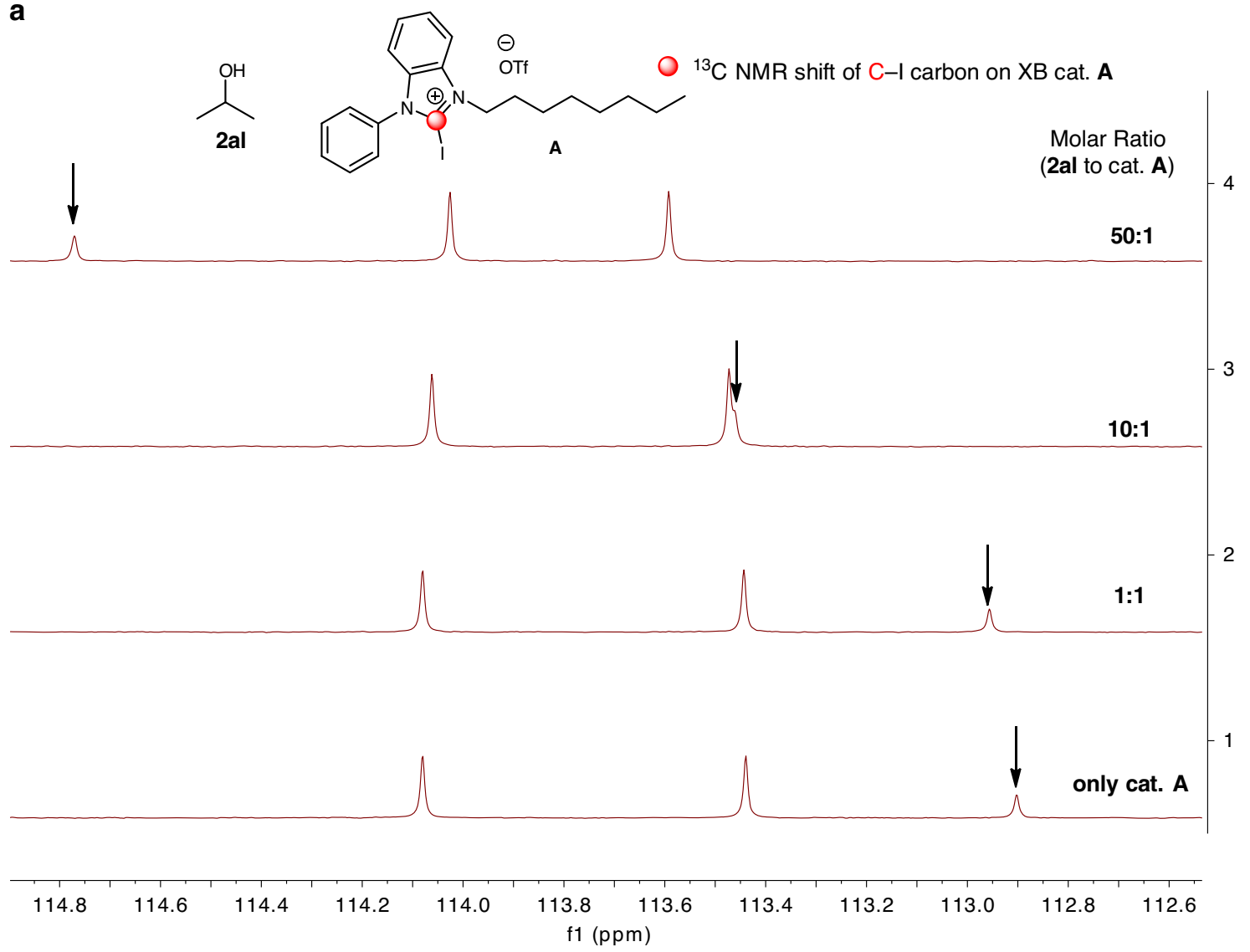

b

${ }^{13} \mathrm{C}$ NMR titration

Fitting of titration curve to a $1: 1$ binding isotherm

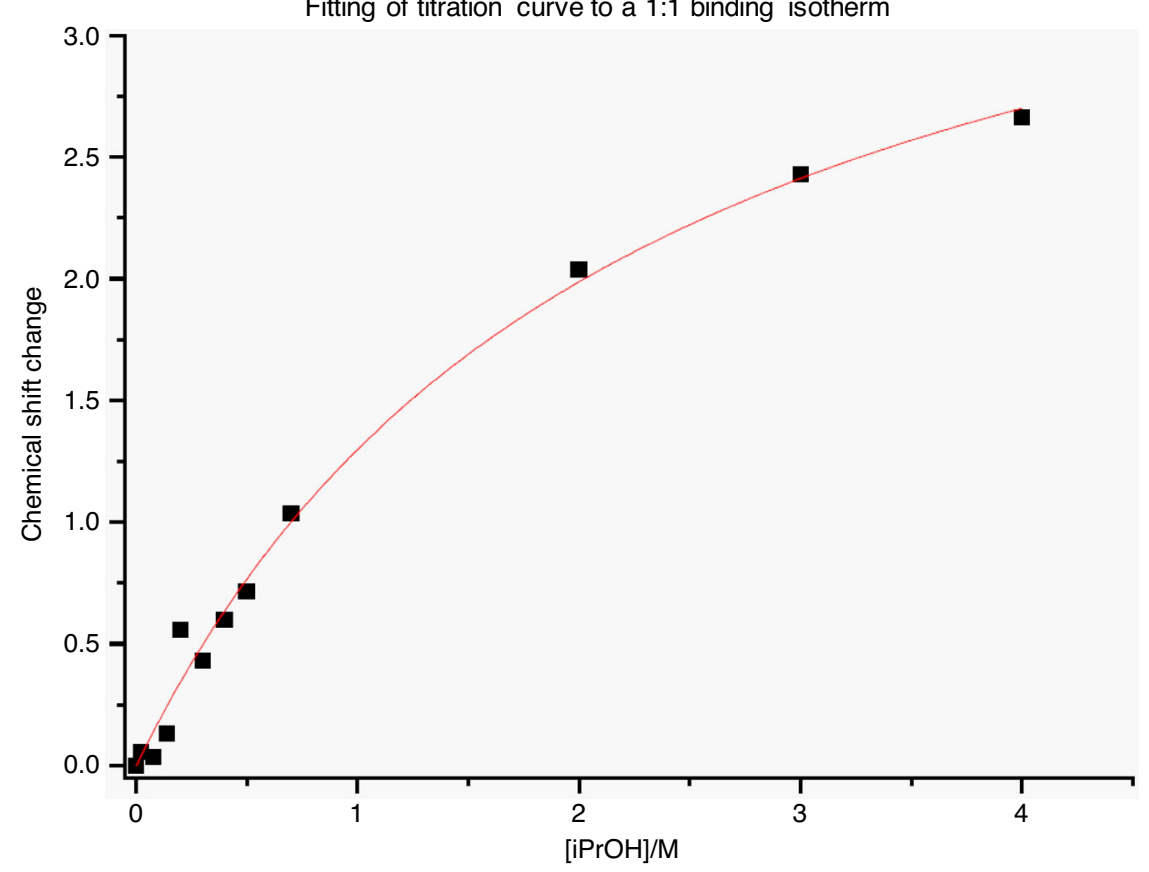

Fig. 4 NMR titrations and isothermal titration calorimetry to ascertain XB activation. a ${ }^{13} \mathrm{C} N \mathrm{MR}$ titration $\left({ }^{13} \mathrm{C}\right.$ resonance of $\mathrm{C}-\mathrm{I}$ bond denoted in arrows). b Fit of the ${ }^{13} \mathrm{C}$ NMR titration to a 1:1 binding isotherm.

product formation profile of $\mathbf{3 a l}$ with the depletion profiles of both reactants (Fig. 6a), isopropanol 2al (limiting reagent) and 1c uncovered the symmetrical nature of the product vs substrates profiles. Previous literature precedence corroborate that such a symmetrical profile is indicative of a slow in situ generation of a critical active catalytic intermediate ${ }^{83}$. Spurred by this finding, we wanted to delineate the mechanistic differences between an XBcatalyzed case with a true strong Brønsted acid catalyst. 


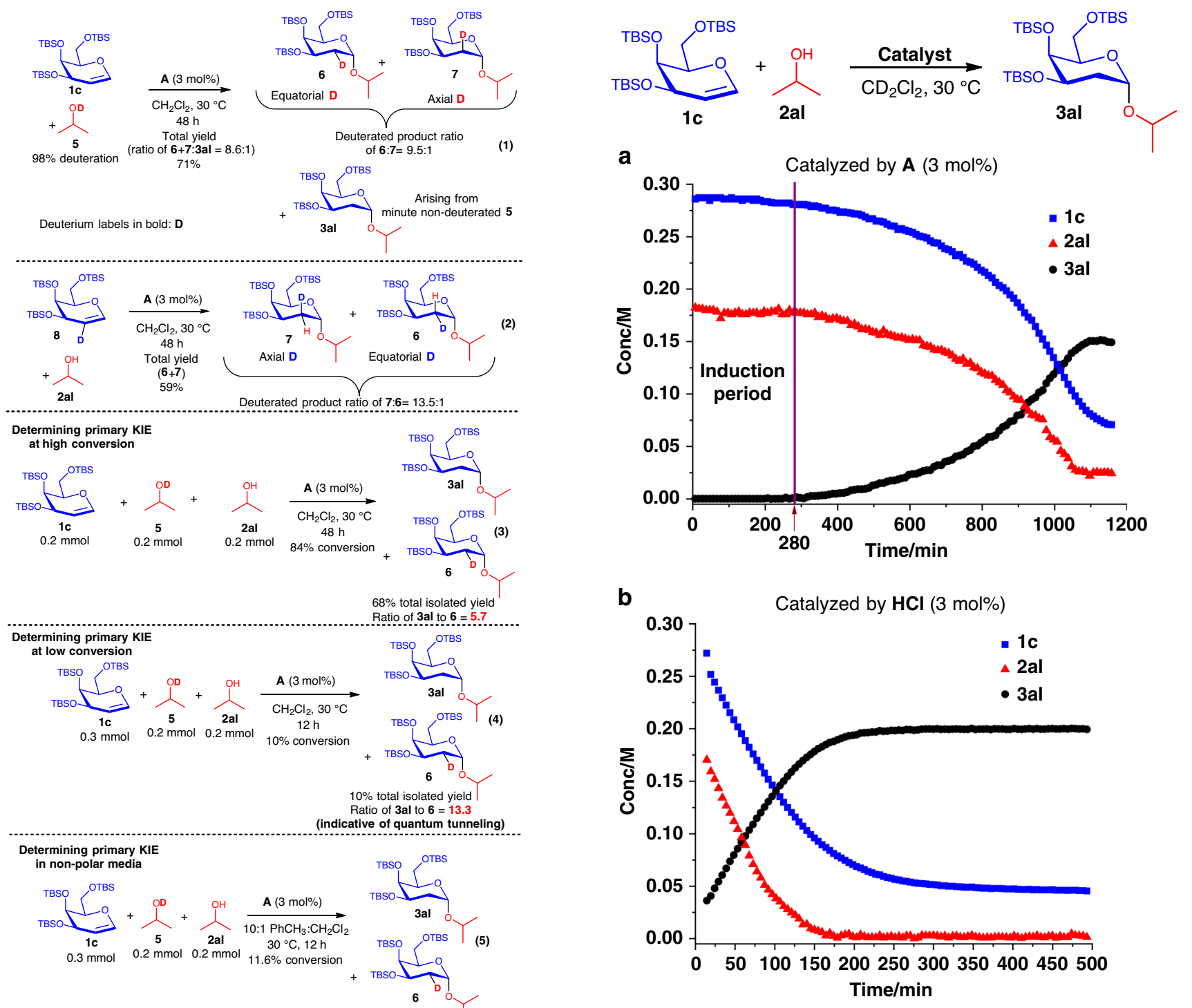

Fig. 6 XB vs strong acid kinetic profile comparison. Comparison of a sigmoidal kinetics under standard conditions with $\mathbf{b}$ saturation kinetics with a strong Brønsted acid.

Comparing the profile of catalyst A with $3 \mathrm{~mol} \% \mathrm{HCl}$ (Fig. 6b) showed clear divergences. True Brønsted acid catalysis adheres to saturation kinetics, and no induction time was observed. This further indicate that in our XB catalytic method, a mechanistically different route from a true Brønsted acid catalyst is operative. To ascertain the postulate of in situ generation of an active catalytic intermediate during the induction period, we conducted a sequential control experiment. 2al is first reacted with catalyst A at $30^{\circ} \mathrm{C}$ for $6 \mathrm{~h}$ in the absence of $1 \mathrm{c}$ (Fig. $7 \mathrm{a}$ ), to first generate more of the in situ catalyst in a time period analogous to the induction period. Subsequently, 1c was added with the immediate commencement of NMR monitoring. We observed then the disappearance of the induction period and a significantly accelerated reaction time of $\sim 400 \mathrm{~min}$.

The acceleration arising by conducting the same experiment in

Fig. 5 Deuterium-labeling studies. Deuterated experiments confirming step-wise mechanism through deuterium scrambling and ascertaining source of C2 proton originates from the glycosyl acceptor. Intermolecular competition experiments to understand primary and secondary kinetic isotopic effects (KIEs), and KIE elevation to support quantum tunneling. sequential order supports the generation of an in situ generated active catalyst from $\mathbf{2} \mathbf{a l}$ and $\mathbf{A}$ (Fig. $7 \mathrm{~b}$ depicts accelerated kinetic trace with respect to standard conditions) ${ }^{83}$. The observation of a concave shaped profile indicates continual amplification (active catalyst increasing at an increasing rate) of the in situ catalyst, as the catalytic cycle proceeds upon $1 \mathbf{c}$ addition ${ }^{86}$. In addition, the 

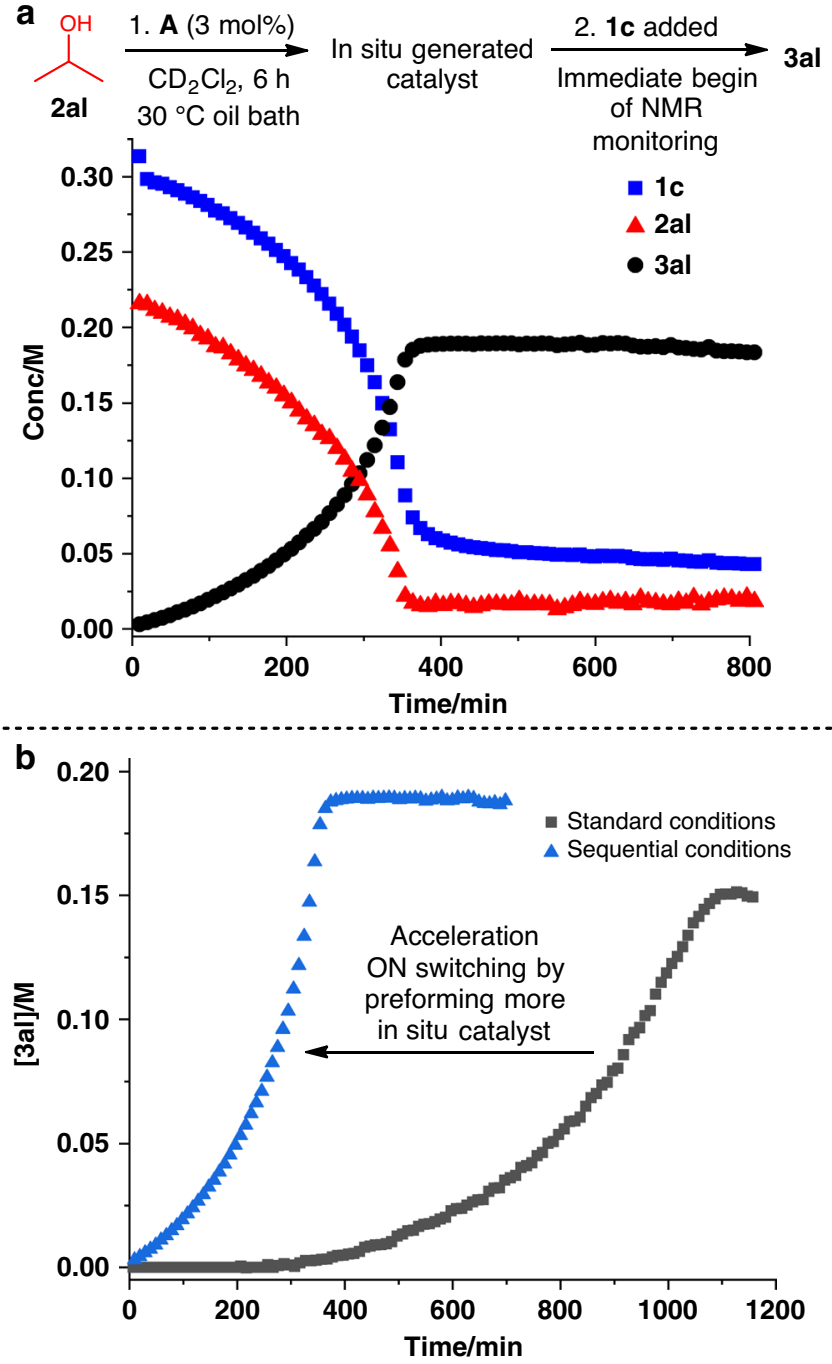

C

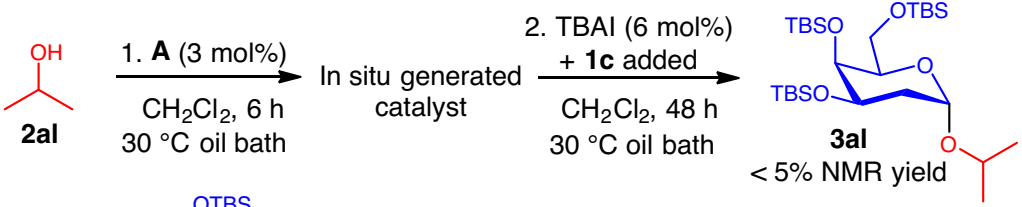

d
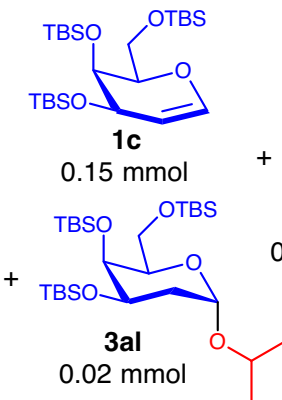

Fig. 7 Sequential and control experiments. a Sequential kinetic experiment unraveled enhanced acceleration of product formation. b Comparison of 3al formation kinetics of standard vs sequential conditions. c Poisoning using TBAI to ascertain XB influence on the in situ catalyst. d Control experiment to support the absence of autocatalysis.

persistently accelerating kinetic behavior also corroborates that the amount of in situ active catalyst present during the entire experiment is significantly lower than the $3 \mathrm{~mol} \%$ of $\mathbf{A}$ added at the onset. This acceleration is analogous to an ON switching of the catalytic network.
In an orthogonal competition experiment to test if this in situ catalytic species involves XB activation, 2al is first reacted with catalyst $\mathbf{A}$ at $30^{\circ} \mathrm{C}$ for $6 \mathrm{~h}$ (Fig. 7c), followed by the sequential addition of TBAI as an in situ XB catalyst poison and $\mathbf{1 c}$ resulted in negligible formation of $\mathbf{3 a l}$. This is consistent with the 
hypothesis that the in situ active catalyst depends crucially on $\mathrm{XB}$ participation for catalytic activity.

To investigate if our sigmoidal kinetic at standard condition is representative of an autoinductive or an autocatalytic profile ${ }^{86-88}$, we conducted a control reaction between 1c, 2al, and product 3al as a putative catalyst in the absence of $\mathbf{A}$. This experiment resulted in negligible conversion $(<5 \%$, Fig. $7 d)$, and further supports the hypothesis of an autoinductive mechanism over autocatalysis ${ }^{83,86}$. Unexpectedly, an in situ NMR monitoring experiment adding 20 mol\% of product 3 al to the standard XB catalytic conditions revealed retardation of catalysis (Fig. 8a). While this observation supports the absence of autocatalytic behavior, it points toward a possible catalyst deactivation by $\mathbf{3 a l}$. It is essential to note that addition of $3 \mathrm{al}$ at the onset of the reaction enables the direct deactivation of $\mathbf{A}$ before the formation of any in situ catalyst in the reaction mixture, hence hampering reaction progression. This retardation effect is analogous to an OFF switching of the catalytic network (Fig. 8b, compared with standard conditions). For a confirmatory test to ascertain XB catalytic influences between 3al and $\mathbf{A}$, an acceptor exchange experiment mixing propargyl alcohol 2r with 3 al in the presence of $\mathbf{A}$ was conducted (Fig. 8c, Supplementary Figs. S49 and 50, and Supplementary Table S10). The temporal 3ak formation and 3al depletion profiles indicated that a XB-catalyzed transacetalization-type reaction is operative, and displayed saturation kinetics. Moreover, this control experiment is indicative of a steady-state dynamic exchange equilibrium between unreacted acceptor in the reaction and the aglycone component of 3al. Intriguingly, evaluation of all NMR spectra along the time coordinate revealed the detection of only $\alpha$ glycosides 3al and 3ak. The absence of $\beta$-glycosides corroborate an $\mathrm{S}_{\mathrm{N}} 1$-type exchange mechanism, which involves the cleavage and reformation of the a-glycosidic linkage. A separate control experiment evaluating transacetalization on benzyl protected Dgalactal revealed that this dynamic acceptor exchange process is not operative when ether protecting groups are used (Supplementary Fig. S55 and Supplementary Discussion 3).

To test the dependence of this dynamic exchange on $\mathrm{XB}$ activation, additional $5 \mathrm{~mol} \%$ TBAI was added into the acceptor exchange experiment conditions to poison A (Fig. 8d). This experiment failed to generate $\mathbf{3 a k}$, supporting crucial XB catalytic influences in this dynamic equilibration. A ${ }^{13} \mathrm{C}$ NMR experiment mixing a 1:1 ratio of A:3al (Fig. 8e) yielded a 0.158 p.p.m. downfield shift of the ${ }^{13} \mathrm{C} \mathrm{C}-\mathrm{I}$ resonance of $\mathbf{A}$, evidencing an $\mathrm{XB}$ catalytic interaction on the glycosidic bond.

Concentration dependence of the reaction profile. In order to understand the mechanistic role of the glycosyl donor, the glycosyl acceptor and the $\mathrm{XB}$ catalyst on the reaction profile, as well a
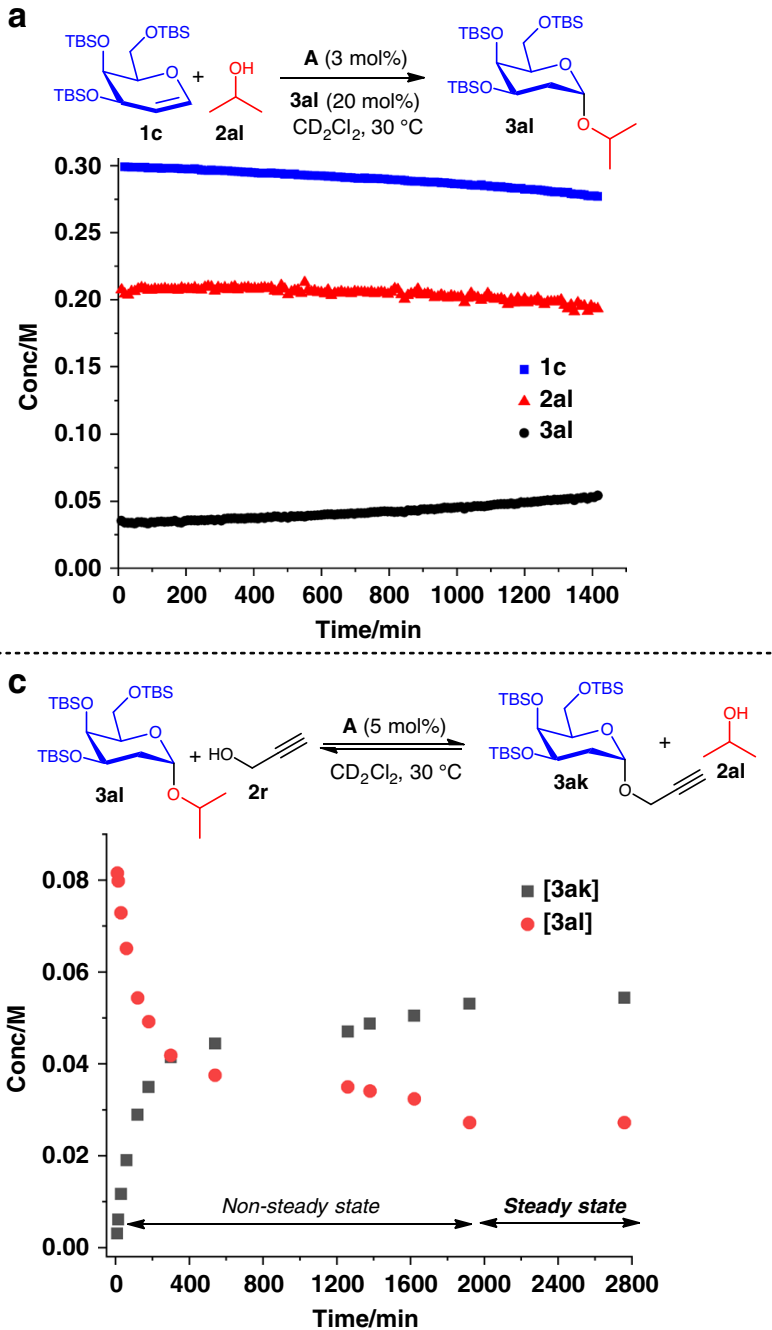

b

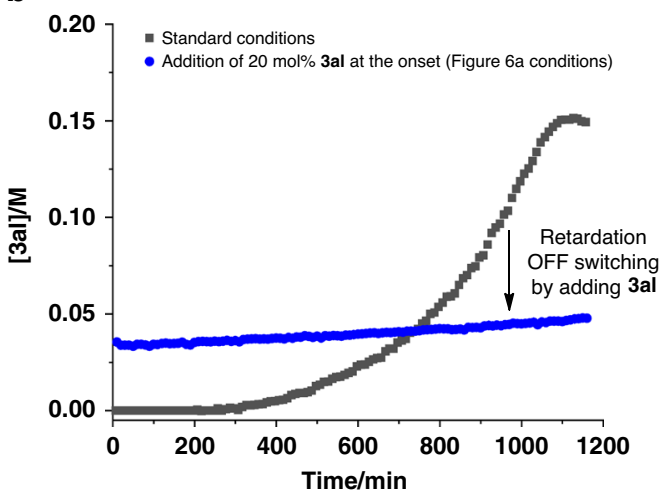

d

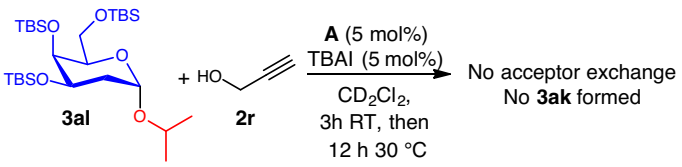

$\stackrel{\ominus}{\ominus}$<smiles></smiles>

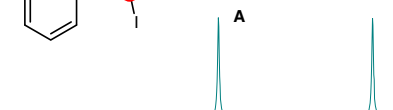

$112.872 \mathrm{ppm}$

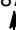
Only A

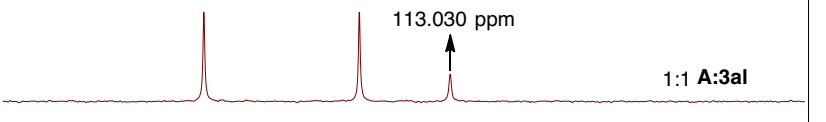

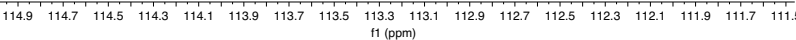

Fig. 8 Dynamic acceptor exchange. a Addition of 20 mol\% product at the onset retarded XB catalysis. $\mathbf{b}$ Comparison of 3 al formation kinetics of standard vs conditions in a. c Acceptor exchange experiment to ascertain dynamic acceptor exchange. d Competition experiment with TBAI showed XB catalyst poisoning halted the dynamic acceptor exchange. e 1:1 adduct of catalyst $\mathbf{A}$ to 3 al revealed a downfield ${ }^{13} \mathrm{C}$ NMR shift. 

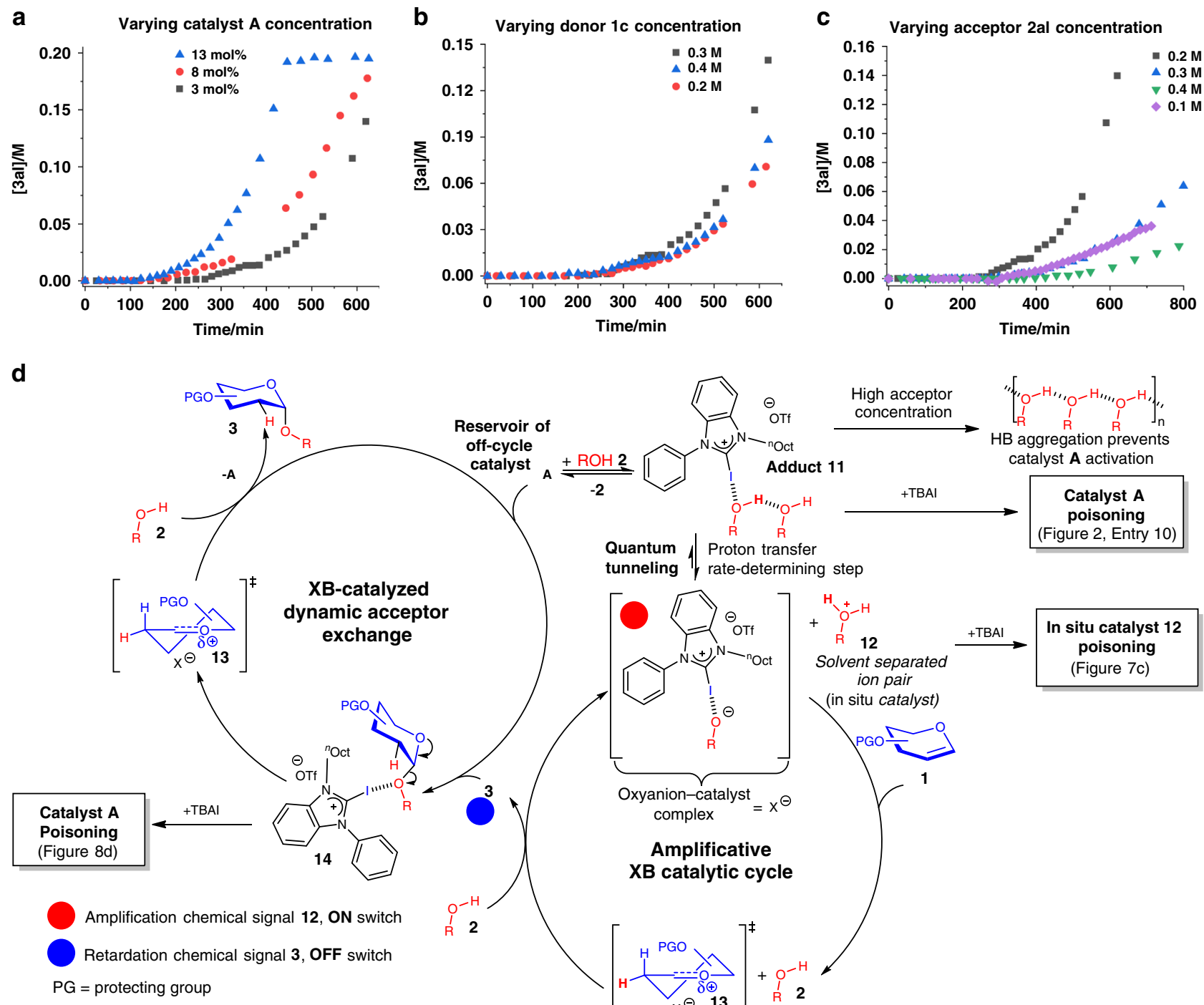
tunneling rate-determining step 
the actual catalyst responsible for product amplification. While we made rigorous efforts to detect this intermediate in situ, however, due to minute amounts of this intermediate (presumably much less than the $3 \mathrm{~mol} \%$ of $\mathbf{A}$ inferred from continuous amplification in standard and sequential kinetic studies) beyond the NMR detection limit (5\%), we are unable to directly detect and characterize the intermediate.

Despite this, based on holistic analysis of numerous informative indirect experiments, such as in situ sequential experiments, base addition, and poisoning of the in situ generated species by TBAI, we deduced that this intermediate possesses Brønsted acidic characteristics, with concurrent XB catalytic dependency. Significantly, TBAI poisoning characteristics were not observed in control true Brønsted acid-catalyzed experiments, such as HOTf, which performed similarly regardless of the presence or absence of TBAI, hence excluding non-XB effects in our mechanism, such as trace acid catalysis ${ }^{21}$.

Hence, we propose based on these data that the catalytic intermediate in our protocol is a solvent-separated ion-pair 12, comprising of a solvated XB-stabilized oxyanion-catalyst complex $\left(\mathrm{X}^{-}\right)$and a solvated protonated acceptor, arising from the rate-determining proton transfer step, which involves quantum tunneling (Fig. 9, and Supplementary Notes 17 and 18). $\mathrm{X}^{-}$will further serve as an oxocarbenium neutralizing counteranion in subsequent steps. Our experiments ascertaining the presence of acceptor $\mathrm{OH}$ bond cleavage (Fig. 5), the crucial involvement of Brønsted acidity (Fig. 2, entries 13-15), and successful TBAI poisoning of the in situ catalyst (Fig. 7c) collectively corroborate the intermediacy of XB-dependent ion-pair 12, as the true in situ catalyst through a proton transfer rate-determining step. This in situ solvent-separated ion-pair hypothesis is also further supported by primary KIE elevation due to increase in the tunneling effect ${ }^{81,82}$, when the reaction was conducted in a more nonpolar 10:1 $\mathrm{PhCH}_{3}: \mathrm{CH}_{2} \mathrm{Cl}_{2}$ solvent mixture. This proton transfer arising from the Lewis acidity of the $\mathrm{XB}$ donor on the alcoholic glycosyl acceptor is reminiscent of the Lewis acidassisted Brønsted acid concept proposed by Yamamoto et al. ${ }^{91}$. In addition, this concept has also been applied by Schmidt et al. in glycosylations using gold complexes or boronates as Lewis acids ${ }^{92,93}$.

12 can subsequently protonate $\mathrm{C} 2$ of glycal $\mathbf{1}$ to form oxocarbenium intermediate 13. This step-wise mechanism is evidenced by scrambling of deuterium labels originating from the acceptor on C2 (Fig. 5, Equations 1 and 2). As 12 gets consumed in the initial catalytic cycle, the reversible $\mathbf{1 1}$ to $\mathbf{1 2}$ reaction will be increasingly shifted toward $\mathbf{1 2}$ as reaction time progresses, in situ amplifying the active catalyst 12 , resulting in an accelerating concave shaped kinetic in the initial phase of the sigmoidal profile (Fig. 7b). Subsequently, the alkoxide in $\mathbf{1 3}$ will attack the oxocarbenium species to form 2-deoxyglycoside 3 with the release of $\mathbf{A}$, coupled with a sequential in-cycle intermolecular proton transfer between two molecules of acceptor 2 under the activation of the released catalyst $\mathbf{A}$ to regenerate the in situ catalyst 12, analogous to an $\mathbf{1 1}$ to $\mathbf{1 2}$ conversion. On the other hand, the formation of more glycoside $\mathbf{3}$ through the amplification cycle (blue circle, Fig. 9d) activates the dynamic exchange mechanism, which siphons off-cycle $\mathbf{A}$, arising from $\mathrm{XB}$ interactions with $\mathbf{A}$ and the glycosidic bond oxygen in $\mathbf{3}$ to form 14. This lowering of the off-cycle concentration of $\mathbf{A}$ due to the dynamic acceptor exchange will accordingly deplete the concentration of $\mathbf{A}$ required to form increasing 12 in the amplificative cycle. This effect is registered by the rate deceleration after the inflection point in the standard sigmoidal kinetic profile (Fig. 6a), but attenuated in the sequential profile (Fig. 7b) due to pre-generation of more 12. However, this effect is strengthened when we directly introduced the glycoside at the onset of the reaction, resulting in retardation (Fig. 8b).
Subsequently, the XB catalytic effect of $\mathbf{A}$ in $\mathbf{1 4}$ results in a dynamic acceptor exchange evidenced by our acceptor exchange experiment (Fig. 8c), TBAI poisoning, and ${ }^{13} \mathrm{C}$ NMR shift (Fig. 8d, e), which involves firstly cleavage of the newly formed glycosidic linkage in $\mathbf{3}$ to reform oxocarbenium intermediate $\mathbf{1 3}$. Next, a new molecule of $\mathbf{2}$ will first initiate a proton transfer with the original alkoxide ion in $\mathrm{X}^{-}$to form a new alkoxide, which will attack the oxocarbenium ion to regenerate 3 coupled with the exit of the original protonated alkoxide as $\mathbf{2}$, releasing catalyst $\mathbf{A}$ back into the off-cycle reservoir. Intriguingly, our proposed mechanism seems to constitute a simplified synthetic mimetic of complex noncovalent interactions modulated biochemical mechanisms catalyzed by enzymes, operating in tightly regulated mechanisms. Unexpectedly, we determined that dynamic XB-modulated mechanistic complexity ${ }^{94}$ could be constructed simply from a ternary chemical mixture comprising of a glycosyl donor, an acceptor, and a XB catalyst.

\section{Benchmarking noncovalent organocatalytic robustness between} $\mathrm{XB}$ and thiourea catalysis. To evaluate the advances in noncovalent catalyst robustness of benzimidazolium XB catalyst $\mathbf{A}$, and we sought to benchmark our $\mathrm{XB}$ catalytic protocol with conventional thioureas $47,56,95,96$.

In an effort to understand broadness and robustness across glycal donors beyond widely reported galactals, we observed a general superiority trend in using XB catalysis over thiourea on these non-galactal substrates. First, within the hexose family, donors such as silylated D-glucals and L-rhamnals work excellently with catalyst $\mathbf{A}$, but not with 15 and 16. Recognizing the possible caveat that could arise from protecting group influences, we performed the comparative glycosylation also with benzylated glucals and rhamnals. Significantly, literature known ether protecting group preference using thiourea catalysis on 2deoxygalactosylation ${ }^{47}$ holds neither for glucosylation nor rhamnosylation.

Second, analogous trends favoring $\mathrm{XB}$ over thiourea catalysis can also be observed from the pentose family of glycal donors. Using silylated D-xylal 1i (Fig. 10c), the benchmarking experiments revealed that catalyst $\mathbf{A}$ enabled smooth glycosylation, but no reaction was observed with 15 or 16. Using a challenging sterically hindered tertiary alcohol, such as $\mathbf{2 a f}$, we noticed that $\mathrm{XB}$ catalysis still furnished the desired glycosylation product smoothly, whereas catalysis via $\mathbf{1 5}$ or $\mathbf{1 6}$ do not generate any observable product. In the case of silylated D-ribal $\mathbf{1 j}$ (Fig. 10d), both catalyst $\mathbf{A}$ and in particular the brominated version of catalyst A (Br-A in parenthesis) gave superior yields superceding the thiourea congeners. This halogen swapping advantage by XB catalysis will be further discussed in a later section. As a control experiment to clarify specificity of protecting group effects on noncovalent catalysis, we noticed that benzylated xylals and ribals remained lowly reactive, regardless whether thiourea or $\mathrm{XB}$ catalysis are employed.

To provide fairer comparison with catalyst 15 and 16, and a more holistic understanding across these noncovalent catalysts, we reproduced the exact conditions reported by Galan and McGarrigle with catalyst A on benzylated galactals, a known strength of these prior methodologies 47,56 . Our comparative data confirmed that ether protected galactals are better suited for thiourea catalysis, although $\mathrm{XB}$ catalysis can also activate benzylated galactals reasonably (Fig. 10e). However, we have demonstrated that employing silyl protecting group in conjunction with $\mathrm{XB}$ catalysis gave consistently excellent performance in yields and anomeric selectivity on the galactal donor family (Fig. 3). In cases where more challenging acceptors, such as the phenolic group in protected tyrosine and isopropanol are used, 
a

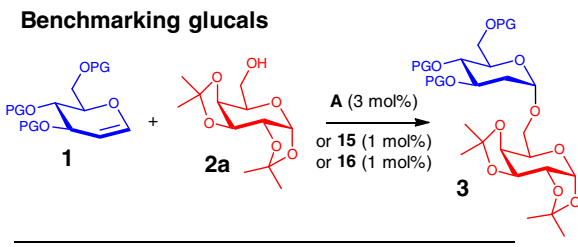

\begin{tabular}{ccc}
\hline $\mathrm{PG}=$ & Silyl (donor 1b) & $\mathrm{Bn}$ \\
Cat. 15 & $<5 \%{ }^{[\mathrm{a}]}$ & $44 \%, \alpha: \beta 76: 24^{[\mathrm{a}]}$ \\
Cat. 16 & $<5 \%{ }^{[\mathrm{a}]}$ & $64 \%, \alpha: \beta 77: 23^{[\mathrm{a}]}$ \\
A & $89 \%, \alpha: \beta>20: 1^{[\mathrm{b}]}$ & $64 \%, \alpha: \beta 75: 25^{[\mathrm{a}]}$
\end{tabular}

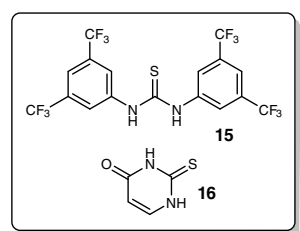

b Benchmarking rhamnals

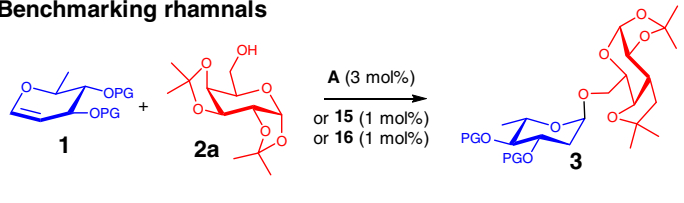

$\begin{array}{ccc}\text { PG }= & \text { Silyl (donor 1f) } & \mathrm{Bn} \\ \text { Cat. 15 } & <5 \%{ }^{[\mathrm{a}]} & 78 \%, \alpha: \beta 83: 17^{[\mathrm{a}]} \\ \text { Cat. 16 } & <5 \%{ }^{[\mathrm{a}]} & 39 \%, \alpha: \beta 87: 13^{[\mathrm{a}]} \\ \text { A } & 88 \%, 88: 12 \alpha: \beta^{[\mathrm{b}]} & 83 \%, \alpha: \beta 86: 14^{[\mathrm{a}]}\end{array}$
C

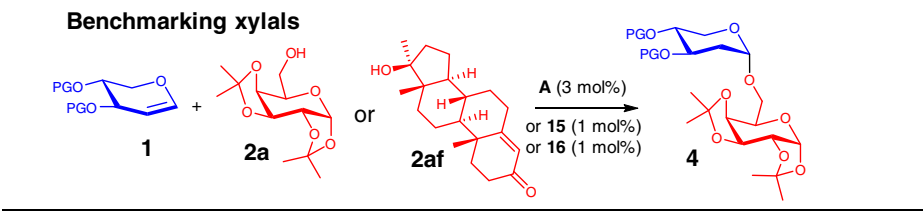

$P G=$ Silyl (donor $1 \mathbf{i}+\mathbf{2 a})$

Cat. $15<5 \%[$ [a]

Cat. $16<5 \%$ [a]

A

$86 \%, \alpha: \beta 83: 17^{[b]}$

Silyl (donor $1 \mathrm{i}+$ tertiary alcohol 2

$<5 \%$ [a]

$<5 \%$ [a]

e

Benchmarking exact 2-deoxygalactosylation substrates reported by Galan and McGarrigle
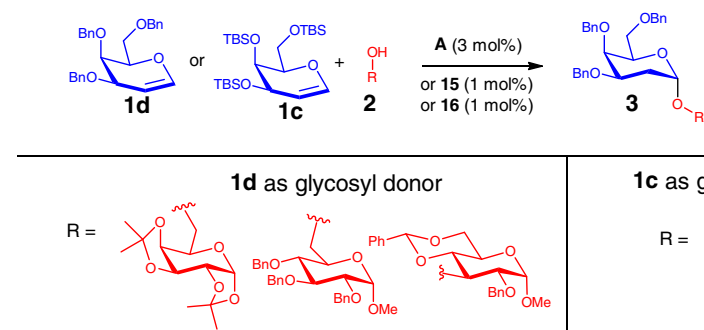

$\begin{array}{cc}\text { Cat. 15 } & 96 \% \\ \text { (lit values) } & \alpha: \beta>20: 1 \\ \text { Cat. 15 } & 94 \%[\text { [b] } \\ \text { (reproduced) } & \alpha: \beta>20: 1 \\ \text { Cat. 16 } & 95 \% \\ \text { (lit values) } & \alpha: \beta>20: 1 \\ \text { Cat. 16 } & 96 \%[b] \\ \text { (reproduced) } & \alpha: \beta>20: 1 \\ \text { A } & 55 \%[b] \\ & \alpha: \beta 85: 15\end{array}$

$\begin{array}{cc}98 \% & 96 \% \\ \alpha: \beta>20: 1 & \alpha: \beta>20: 1 \\ 93 \%[b] & 72 \%[b] \\ \alpha: \beta>20: 1 & \alpha: \beta>20: 1 \\ \text { Not } & 89 \% \\ \text { reported } & \alpha: \beta>20: 1 \\ & 72 \% \%^{[b]} \\ - & \alpha: \beta>20: 1 \\ 70 \%[b] & 46 \%[c] \\ \alpha: \beta>20: 1 & \alpha: \beta>20: 1\end{array}$

$\alpha: \beta>20: 1 \quad \alpha: \beta>20: 1$ 1c as glycosyl donor

$$
\mathrm{R}=
$$

$\mathrm{R}=$

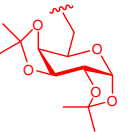

Cat. 15

(lit values) $\quad \begin{aligned} & 72 \%[b] \\ & {[b>20}\end{aligned}$

Cat. $15 \quad 68 \%[b]$

(reproduced) $\alpha: \beta>20: 1$

Cat. $16 \quad 11 \%[d]$

(lit values) $\quad \alpha: \beta>20: 1$

Cat. 16

(reproduced) $<5 \%[a, d]$

A $\quad 91 \% \%^{[b]}$ d
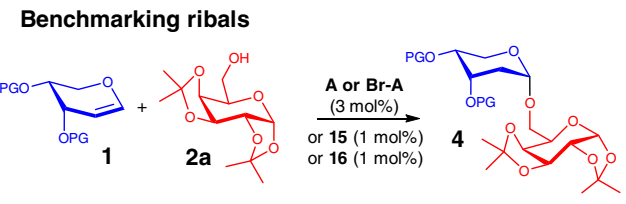

$P G=$

Silyl (donor $\mathbf{1} \mathbf{j}+\mathbf{2 a}$ )

$\mathrm{Bn}$

Cat. 15

$44 \%,>20: 1 \alpha: \beta^{[b]}$

$<5 \%[c]$

Cat. 16

$52 \%,>20: 1 \alpha: \beta^{[b]}$

$<5 \%$ [c]

A $(\mathrm{Br}-\mathrm{A}) \quad 64 \%(81 \%),>20: 1 \alpha: \beta^{[\mathrm{b}]} \quad<5 \%[\mathrm{c}]$

Fig. 10 Benchmarking XB and thiourea organocatalysis. [a] ${ }^{1} \mathrm{H}$ NMR yield determined with 1,3,5-trimethoxybenzene as an internal standard. [b] Isolated yields after flash column chromatography. [c] A mixture with inseparable Ferrier-type product impurity was obtained after flash chromatography purification, the yield was calculated by ${ }^{1} \mathrm{H}$ NMR analysis. [d] Reaction time was $48 \mathrm{~h}$, literature gave $68 \%$ yield with 2 -methyl THF as solvent at $83{ }^{\circ} \mathrm{C}$ refluxing temperature. a Benchmarking glucals, b Benchmarking rhamnals, c Benchmarking xylals, d Benchmarking ribals, e Benchmarking reported 2deoxygalactosylation substrates, $\mathbf{f}$ Benchmarking challenging 2-deoxygalactosylation acceptors.

$\mathrm{XB}$ catalysis gave clear superiority, while thiourea/thiouracil protocols generated negligible product (Fig. 10f).

In all, we note that $\mathrm{XB}$ catalysis offered distinct advantages over thiourea/thiouracil catalysis in activating the entire spectrum of different sugar donors beyond galactals. Pure thiourea catalysis is useful in 2-deoxygalactosylation with ether protecting groups, as previously demonstrated by Galan and McGarrigle et al. ${ }^{47,56,95,96}$, but reactivity do not scale well across donors, as well as challenging acceptors, and the limitations are evident when other non-galactal glycosyl donors are employed.

These benchmarking experiments reinforce immense value of exploiting $\mathrm{XB}$ catalytic protocols as an enhanced organocatalytic tool for accommodating a broader variety of non-galactal glycosyl donors, when conventional thiourea catalytic methods are not optimal.

Applicability of switchable mechanism to elevate yields. To demonstrate further applicability of the XB-dependent "switchable" characteristic, we performed sequential experiments analogous to Fig. 7 in an attempt to elevate yields of representative rhamnosides 3aq and 3at (Fig. 11a) that gave moderate yields in our reaction scope under standard conditions. To our delight, the pre-generation of in situ catalysts had a positive effect in our protocol to substantially improve chemical yields of sluggish substrates. 
a
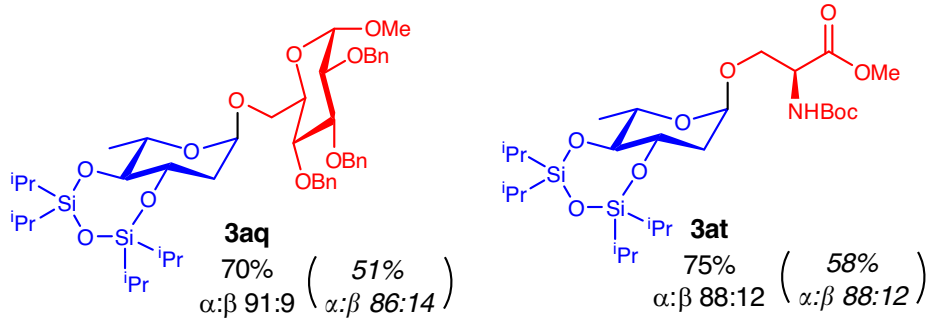

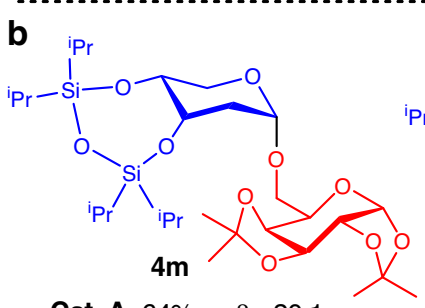

Cat. A: $64 \%, \alpha: \beta>20: 1$

Cat. Br-A: $81 \%, \alpha: \beta>20: 1$

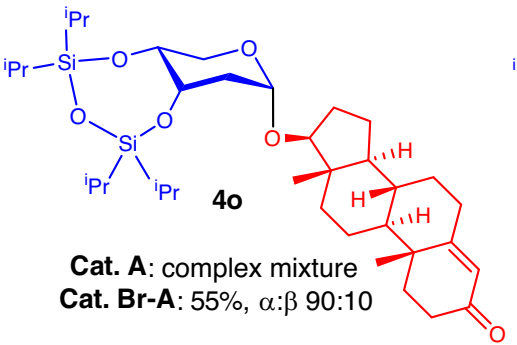<smiles>CCCCC(N)C(=O)OC[C@H](N)C(=O)OC</smiles>

Cat. A: complex mixture Cat. Br-A: $71 \%, \alpha: \beta>20: 1$

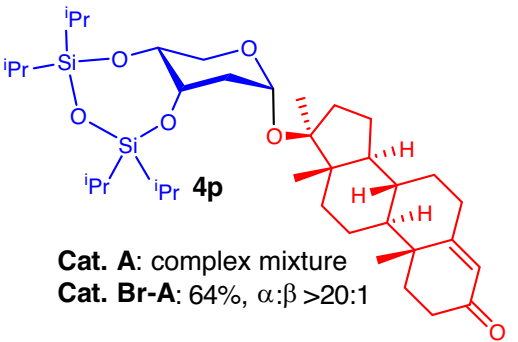

Fig. 11 XB tunability. a Elevation of yields on rhamnosides using the sequential procedure, yields, and selectivity from standard conditions indicated in parenthesis. $\mathbf{b}$ lodine to bromide halogen swapping on $\mathbf{A}$ resulted in significantly expanded 2-deoxyribosylation scope.

Tunability of XB catalysis via halogen swapping. Despite the broadly improved performance of catalyst $\mathbf{A}$, we noticed certain limitations of our protocol utilizing the iodide derivative $\mathbf{A}$, particularly in 2-deoxyglycosylation reactions with D-ribals. Meticulous experiments revealed that $\mathbf{A}$ is very sensitive to the acceptors employed, furnishing complex mixtures when aminoacid-derived acceptors or more sterically hindered secondary and tertiary alcohol containing steroidal acceptors were employed (Fig. 11b, 4n-4p).

To address this issue, we reasoned that the $\sigma$-hole on the iodine atom on $\mathbf{A}$ could be fine-tuned by employing an iodine to bromine halogen swapping strategy. Surprisingly, using the bromide derivative $\mathbf{B r}-\mathbf{A}$ as catalyst showed marked improvement (Fig. 11b) to access such challenging glycosidic linkages in $\mathbf{4 n - 4 p}$ with very good to excellent anomeric selectivity, which were not accessible via $\mathbf{A}$.

Gratifyingly, even 2-deoxyribosylation to $\mathbf{4 m}$ gave substantially improved yield (81\%) using Br-A. These observations support that postulate that stronger iodide $\mathrm{XB}$ donors might be overly strong Lewis acids for sensitive ribal substrates, which resulted in undesired decomposition pathways. As such, tuning $\mathrm{XB}$ donor strength by employing the weaker Br-A donor could provide an attenuated, milder catalytic activation route which circumvents decomposition, but increase catalytic performance. This success of our halogen swapping method depicts the power of $\mathrm{XB}$ catalysis as a high-performance-tunable noncovalent organocatalytic tool, especially when sensitivity of substrates is pivotal.

\section{Discussion}

In conclusion, we demonstrate a robust $\mathrm{XB}$ organocatalyzed 2deoxyglycosylation, operating through an intricately $\mathrm{XB}$ catalyst controlled reaction network. This report showcases a marked advancement in noncovalent organocatalysis, where the use of lesser explored XB catalysis resulted in mechanistic divergences, and significantly elevated substrate scope compared to conventional thiourea catalysis, particularly in the formation of challenging glycosidic linkages. Detailed mechanistic studies were also illuminating and instructive of unique and hitherto unknown intricacies and dynamism of XB activation, such as the involvement of quantum tunneling.

In situ temporal kinetic experiments and comparison revealed a sigmoidal kinetic profile characteristic of autoinductive reactions. KIE measurements also revealed unusually high KIE values beyond the semiclassical limit, as well as KIE elevation in nonpolar solvents, which are indicative of quantum tunneling and the formation of an in situ ion-pair intermediate in the ratedetermining proton transfer step. Intriguingly, deeper studies unraveled a biomimetic reaction network embedded with switchable ON/OFF mechanism, modulated by dynamic XB interactions between catalyst, substrates, and products. Furthermore, we capitalized on this switchable property, to elevate yields of 2-deoxyrhamnosides through a sequential protocol. We also demonstrate that tuning $\sigma$-hole properties via a halogen swap on the catalyst is beneficial in elevating $\mathrm{XB}$ catalyst robustness on glycosyl donors, such as D-ribals, particularly when more challenging glycosyl acceptors are employed.

We are optimistic that the development of this protocol has promising wider implications in noncovalent organocatalysis, in deconvoluting differences of mechanism and substrate tolerances between XB and thiourea catalysis, and will contribute to developing mild XB catalysis as a competitive new generational tool in challenging, but biologically relevant bond forming reactions. 


\section{Methods}

General techniques. Unless otherwise stated, all reactions were set up under inert atmosphere (argon) utilizing glassware that were oven-dried and cooled under argon purging. Silica gel flash column chromatography was performed on deactivated silica gel Merck 60 (particle size $40-63 \mu \mathrm{m}$ ). Starting materials were procured from commercial sources and used without purifications. Solvents were dried according to reported procedures or procured from commercial suppliers. Monitoring of reactions was done using thin layer chromatography (TLC) on Merck silica gel aluminum plates with F254 indicator. TLC visualization of plates was performed under UV light $(254 \mathrm{~nm})$ or employing $\mathrm{KMnO}_{4}$ stain or $\mathrm{H}_{2} \mathrm{SO}_{4}$-EtOH $\left(10 \% \mathrm{H}_{2} \mathrm{SO}_{4} \mathrm{v} / \mathrm{v}\right)$

NMR spectra were collected at $300 \mathrm{~K}$ on a Bruker DRX400 $(400 \mathrm{MHz})$, Bruker DRX500 (500 MHz), INOVA500 (500 MHz), or Bruker DRX700 (700 MHz) spectrometers, using $\mathrm{CDCl}_{3}, \mathrm{CD}_{2} \mathrm{Cl}_{2}$, acetone-d6, $\mathrm{CD}_{3} \mathrm{CN}$, or benzene-d6 as deuterated solvents. Data for ${ }^{1} \mathrm{H}$ NMR are reported as follows: chemical shift $(\delta \mathrm{p}$. p.m.), multiplicity ( $\mathrm{s}=$ singlet, $\mathrm{d}=$ doublet, $\mathrm{t}=$ triplet, $\mathrm{q}=$ quartet, $\mathrm{m}=$ multiplet, and $\mathrm{br}=$ broad), coupling constant $(\mathrm{Hz})$, NMR spectra were internally referenced to the following residual solvent signals $\left(\mathrm{CDCl}_{3}: \delta=7.26\right.$ p.p.m. for ${ }^{1} \mathrm{H}, \delta=77.16$ p.p.m. for ${ }^{13} \mathrm{C} ; \mathrm{CD}_{2} \mathrm{Cl}_{2}: \delta=5.32$ p.p.m. for ${ }^{1} \mathrm{H}, \delta=54.00$ p.p.m. for ${ }^{13} \mathrm{C}$; acetoned6: $\delta=2.05$ p.p.m. for ${ }^{1} \mathrm{H}, \delta=29.92$ p.p.m. for ${ }^{13} \mathrm{C}$; $\mathrm{CD}_{3} \mathrm{CN}$ : $\delta=1.94$ p.p.m. for ${ }^{1} \mathrm{H}, \delta=1.32$ p.p.m. for ${ }^{13} \mathrm{C}$ of $\mathrm{CD}_{3}, \mathrm{C}_{6} \mathrm{D}_{6}: \delta=7.16$ p.p.m. for ${ }^{1} \mathrm{H}, \delta=128.06$ p.p.m. for ${ }^{13} \mathrm{C}$ ).

High-resolution mass spectra were recorded on an LTQ Orbitrap mass spectrometer coupled to an Accela HPLC-System (HPLC column: Hypersyl GOLD, $50 \mathrm{~mm} \times 1 \mathrm{~mm}$, particle size $1.9 \mu \mathrm{m}$, ionization method: electron spray ionization) and Bruker ultrafleXtreme MALDI-TOF-TOF (three decimal accuracy). Optical rotations were measured in a Schmidt + Haensch Polartronic $\mathrm{HH} 8$ polarimeter equipped with a sodium lamp source $(589 \mathrm{~nm})$, and are reported as follows: $[\alpha] D T^{\circ} \mathrm{C}(c=\mathrm{g} / 100 \mathrm{~mL}$, solvent). ITC experiments were performed on a MicroCal VP-ITC device.

The anomeric ratio was determined by ${ }^{1} \mathrm{H}$ NMR spectroscopy on the crude reaction mixture and the anomeric configuration was determined by $2 \mathrm{D}$ NOESY. Chemical yields refer to combined isolated yields of all anomers after flash column chromatography. NMR yields were determined using appropriate internal standards.

General procedure for XB-catalyzed 2-deoxyglycosylation. A mixture of catalyst A (3-10 mol\%), glycosyl acceptor 2 ( $0.2 \mathrm{mmol})$, and glycal $\mathbf{1}(0.3-0.4 \mathrm{mmol})$ was dissolved in anhydrous $\mathrm{CH}_{2} \mathrm{Cl}_{2}(1 \mathrm{~mL}, 0.2 \mathrm{M}$ for 2$)$, and sealed in a dry tube under argon. The mixture was stirred at a specific temperature (room temperature to $50{ }^{\circ} \mathrm{C}$ depending on the substrate). Subsequently, the solvent was removed under reduced pressure and the residue was first analyzed by crude ${ }^{1} \mathrm{H}$ NMR to determine anomeric selectivity and then subjected to flash column chromatography (dry loading) to give the 2-deoxyglycoside 3 or 4 .

\section{Data availability}

The authors declare that the data supporting the findings of this study are available within the article and its Supplementary Information files. Additional data are available from the corresponding author upon reasonable request.

Received: 14 January 2020; Accepted: 26 August 2020;

Published online: 30 September 2020

\section{References}

1. Knowles, R. R. \& Jacobsen, E. N. Attractive noncovalent interactions in asymmetric catalysis: links between enzymes and small molecule catalysts. Proc. Natl Acad. Sci. USA 107, 20678-20685 (2010).

2. Marchetti, L. \& Levine, M. Biomimetic catalysis. ACS Catal. 1, 1090-1118 (2011).

3. Wheeler, S. E., Seguin, T. J., Guan, Y. \& Doney, A. C. Noncovalent interactions in organocatalysis and the prospect of computational catalysis design. Acc. Chem. Res. 49, 1061-1069 (2016).

4. Mattson, A. E. Tricks for noncovalent catalysis. Science 358, 720 (2017).

5. Schreiner, P. R. Metal-free organocatalysis through explicit hydrogen bonding interactions. Chem. Soc. Rev. 32, 289-296 (2003).

6. Doyle, A. G. \& Jacobsen, E. N. Small-molecule H-bond donors in asymmetric catalysis. Chem. Rev. 107, 5713-5743 (2007).

7. Takemoto, Y. Recognition and activation by ureas and thioureas: stereoselective reactions using ureas and thioureas as hydrogen-bonding donors. Org. Biomol. Chem. 3, 4299-4306 (2005).

8. Connon, S. J. Organocatalysis mediated by (thio)urea derivatives. Chem. Eur. J. 12, 5418-5427 (2006).

9. Zhang, Z. \& Schreiner, P. R. (Thio)urea organocatalysis-what can be learnt from anion recognition. Chem. Soc. Rev. 38, 1187-1198 (2009).
10. Takemoto, Y. Development of chiral thiourea catalysts and its application to asymmetric catalytic reactions. Chem. Pharm. Bull. 58, 593-601 (2010).

11. Serdyuk, O. V., Heckel, C. M. \& Tsogoeva, S. B. Bifunctional primary aminethioureas in asymmetric organocatalysis. Org. Biomol. Chem. 11, 7051-7071 (2013).

12. Tsakos, M. \& Kokotos, C. G. Primary and secondary amine-(thio)ureas and squaramides and their applications in asymmetric organocatalysis. Tetrahedron 69, 10199-10222 (2013).

13. Nishikawa, Y. Recent topics in dual hydrogen bonding catalysis. Tetrahedron Lett. 59, 216-223 (2018).

14. Odagi, M. \& Nagasawa, K. Recent advances in natural products synthesis using bifunctional organocatalysts bearing a hydrogen-bonding donor moiety. Asian J. Org. Chem. 8, 1766-1774 (2019).

15. Beale, T. M., Chudzinski, M. G., Sarwar, M. G. \& Taylor, M. S. Halogen bonding in solution: thermodynamics and applications. Chem. Soc. Rev. 42, 1667-1680 (2013).

16. Cavallo, G. et al. The halogen bond. Chem. Rev. 116, 2478-2601 (2016).

17. Bulfield, D. \& Huber, S. M. Halogen bonding in organic synthesis and organocatalysis. Chem. Eur. J. 22, 14434-14450 (2016).

18. Breugst, M., von der Heiden, D. \& Schmauck, J. Novel noncovalent interactions in catalysis: a focus on halogen, chalcogen, and anion- $\pi$ bonding. Synthesis 49, 3224-3236 (2017).

19. Zhao, Y., Cotelle, Y., Sakai, N. \& Matile, S. Unorthodox interactions at work. J. Am. Chem. Soc. 138, 4270-4277 (2016)

20. Tepper, R. \& Schubert, U. S. Halogen bonding in solution: anion recognition, templated self-assembly, and organocatalysis. Angew. Chem. Int. Ed. 57, 6004-6016 (2018)

21. Sutar, R. L. \& Huber, S. M. Catalysis of organic reactions through halogen bonding. ACS Catal. 9, 9622-9639 (2019).

22. Scholfield, M. R., Vander Zanden, C. M., Carter, M. \& Ho, P. S. Halogen bonding (X-Bonding): a biological perspective. Protein Sci. 22, 139-152 (2013).

23. Bruckmann, A., Pena, M. A. \& Bolm, C. Organocatalysis through halogenbond activation. Synlett 6, 900-902 (2008).

24. Coulembier, O., Meyer, F. \& Dubois, P. Controlled room temperature ROP of L-lactide by $\mathrm{ICl}_{3}$ : a simple halogen-bonding catalyst. Polym. Chem. 1, 434-437 (2010).

25. Walter, S. M., Kniep, F., Herdtweck, E. \& Huber, S. M. Halogen-bond-induced activation of a carbon-heteroatom bond. Angew. Chem. Int. Ed. 50, 7187-7191 (2011).

26. Kniep, F. et al. Organocatalysis by neutral multidentate halogen-bond donors Angew. Chem. Int. Ed. 52, 7028-7032 (2013).

27. Jungbauer, S. H. et al. Activation of a carbonyl compound by halogen bonding. Chem. Commun. 50, 6281-6284 (2010).

28. He, W., Ge, Y.-C. \& Tan, C.-H. Halogen-bonding-induced hydrogen transfer to $\mathrm{C}=\mathrm{N}$ bond with Hantzsch ester. Org. Lett. 16, 3244-3247 (2014).

29. Jungbauer, S. \& Huber, S. M. Cationic Multidentate halogen-bond donors in halide abstraction organocatalysis: catalyst optimization by preorganization. $J$. Am. Chem. Soc. 137, 12110-12120 (2015).

30. Takeda, Y., Hisakuni, D., Lin, C.-H. \& Minikata, S. 2-Halogenoimidazolium salt catalyzed aza-diels-alder reaction through halogen-bond formation. Org. Lett. 17, 318-321 (2015).

31. Saito, M., Tsuji, N., Kobayashi, Y. \& Takemoto, Y. Direct dehydroxylative coupling reaction of alcohols with organosilanes through $\mathrm{Si}-\mathrm{X}$ bond activation by halogen bonding. Org. Lett. 17, 318-321 (2015).

32. Saito, M., Kobayashi, Y., Tsuzuki, S. \& Takemoto, Y. Electrophilic activation of iodonium ylides by halogen-bond-donor catalysis for cross-enolate coupling. Angew. Chem. Int. Ed. 56, 7653-7657 (2017).

33. Chan, Y.-C. \& Yeung, Y.-Y. Halogen bond catalyzed bromocarbocyclization. Angew. Chem. Int. Ed. 57, 3483-3487 (2018).

34. Benz, S., Poblador-Bahamonde, A., Low-Ders, N. \& Matile, S. Cataysis with pnictogen, chalcogen, and halogen bonds. Angew. Chem. Int. Ed. 57, 5408-5412 (2018).

35. Chan, Y.-C. \& Yeung, Y.-Y. Halogen-bond-catalyzed addition of carbon-based nucleophiles to $N$-acylimminium ions. Org. Lett. 21, 5665-5669 (2019).

36. Kuwano, S., Suzuki, T., Hosaka, Y. \& Arai, T. A chiral organic base catalyst with halogen-bonding-donor functionality: asymmetric mannich reactions of malononitrile with $\mathrm{N}$-Boc aldimines and ketimines. Chem. Commun. 54, 3847-3850 (2018).

37. Heinen, F., Engelage, E., Dreger, A., Weiss, R. \& Huber, S. M. Iodine(III) derivatives as halogen bonding organocatalysts. Angew. Chem. Int. Ed. 57, 3830-3833 (2018)

38. Kuwano, S., Suzuki, T., Yamanaka, M., Tsutsumi, R. \& Arai, T. Catalysis based on C-I... $\pi$ halogen bonds: electrophilic activation of 2-alkenylindoles by cationic halogen-bond donor for [4+2] cycloadditions. Angew. Chem. Int. Ed. 58, 10220-10224 (2019).

39. Guillier, K. et al. A halogen-bond donor catalyst for templated macrocyclization. Angew. Chem. Int. Ed. 58, 14940-14943 (2019). 
40. Dreger, A. et al. A halogen-bonding-catalysed nazarov cyclisation reaction. Chem. Commun. 55, 8262-8265 (2019).

41. Liu, X., Ma, S. \& Toy, P. H. Halogen bond-catalyzed Friedel-Crafts reactions of aldehydes and ketones using a bidentate halogen bond donor catalyst: synthesis of symmetrical bis(indolyl)methanes. Org. Lett. 21, 9212-9216 (2019).

42. Kaasik, M. et al. Halo-1,2,3-triazolium salts as halogen bond donors for the activation of imines in dihydropyridinone synthesis. J. Org. Chem. 84, 4294-4303 (2019).

43. Kee, C. W. \& Wong, M. W. In silico design of halogen-bonding-based organocatalyst for Diels-Alder reaction, claisen rearrangement, and cope-type hydroamination. J. Org. Chem. 81, 7459-7470 (2016).

44. Xu, C. \& Loh, C. C. J. A multi-stage halogen bond catalyzed strain-release glycosylation unravels new hedgehog signaling inhibitors. J. Am. Chem. Soc 141, 5381-5391 (2019).

45. Xu, C. \& Loh, C. C. J. An ultra-low thiourea catalyzed strain-release glycosylation and a multicatalytic diversification strategy. Nat. Commun. 9 , 4057 (2018).

46. Bennett, C. S. \& Galan, M. C. Methods for 2-deoxyglycoside synthesis. Chem. Rev. 118, 7931-7985 (2018).

47. Balmond, E. I., Coe, D. M., Galan, M. C. \& McGarrigle, E. M. a-Selective organocatalytic synthesis of 2-deoxygalactosides. Angew. Chem. Int. Ed. 51, 9152-9155 (2012).

48. Balmond, E. I. et al. A 3,4-trans-fused cyclic protecting group facilitates $\alpha$ selective catalytic synthesis of 2-deoxyglycosides. Angew. Chem. Int. Ed. $\mathbf{5 3}$ 8190-8194 (2014).

49. Palo-Nieto, C., Sau, A., Williams, R. \& Galan, M. C. Cooperative Brønsted acid-type organocatalysis fort the stereoselective synthesis of deoxyglycosides. J. Org. Chem. 82, 407-414 (2017).

50. Sau, A. et al. Palladium-catalyzed direct stereoselective synthesis of deoxyglycosides from glycals. Angew. Chem. Int. Ed. 56, 3640-3644 (2017).

51. Palo-Nieto, C., Sau, A. \& Galan, M. C. Gold(I)-catalyzed directed stereoselective synthesis of deoxyglycosides from glycals. J. Am. Chem. Soc. 139, 14041-14044 (2017).

52. Das, S., Pekel, D., Neudörfl, J.-M. \& Berkessel, A. Organocatalytic glycosylation by using electron-deficient pyridinium salts. Angew. Chem. Int. Ed. 54, 12479-12483 (2015).

53. Zhao, G. \& Wang, T. Stereoselective synthesis of 2-deoxyglycosides from glycals by visible-light-induced photoacid catalysis. Angew. Chem. Int. Ed. 57, 6120-6124 (2018).

54. Sherry, B. D., Loy, R. N. \& Toste, F. D. Rhenium(V)-catalyzed synthesis of 2deoxy-a-glycosides. J. Am. Chem. Soc. 126, 4510-4511 (2004).

55. Ghosh, T., Mukherji, A. \& Kancharla, P. K. Sterically hindered 2,4,6-Tri-tertbutylpyridinium salts as single hydrogen bond donors for highly steroselective glycosylation reactions of glycals. Org. Lett. 21, 3490-3495 (2019).

56. Bradshaw, G. A. et al. Stereoselective organocatalyzed glycosylationsthiouracil, thioureas and monothophthalimide act as Brønsted acid catalysts at low loadings. Chem. Sci. 10, 508-514 (2019).

57. Nakatsuji, Y., Kobayashi, Y. \& Takemoto, Y. Direct addition of amides to glycals enabled by solvation-insusceptible 2-haloazolium salt catalysis. Angew. Chem. Int. Ed. 58, 14115-14119 (2019).

58. Palo-Nieto, C. et al. Copper reactivity can be tuned to catalyze the stereoselective synthesis of 2-deoxyglycosides from glycals. Org. Lett. 22, 1991-1996 (2020).

59. Liu, M. et al. Stereoselective electro-2-deoxyglycosylation from glycals. Angew. Chem. Int. Ed. 59, 15204-15208 (2020).

60. Williams, R. \& Galan, M. C. Recent advances in organocatalytic glycosylations. Eur. J. Org. Chem. 2017, 6247-6264 (2017).

61. Park, Y. et al. Macrocyclic bis-thioureas catalyze stereospecific glycosylation reactions. Science 355, 162-166 (2017).

62. Levi, S. M., Li, Q., Rötheli, A. \& Jacobsen, E. N. Catalytic activation of glycosyl phosphates for stereoselective coupling reactions. Proc. Natl Acad. Sci. USA 116, 35-39 (2019)

63. Mayfield, A. B., Metternich, Trotta, A. H. \& Jacobsen, E. N. Stereospecific furanosylations catalyzed by bis-thiourea hydrogen bond donors. J. Am. Chem. Soc. 142, 4061-4069 (2020).

64. Li, Q., Levi, S. M. \& Jacobsen, E. N. Highly stereoselective $\beta$-mannosylations and $\beta$-rhamnosylations catalyzed by bis-thiourea. J. Am. Chem. Soc. 142, 11865-11872 (2020).

65. Overend, W. G., Rees, C. W. \& Sequeira, J. S. Reactions at position 1 of carbohydrates. Part III. The acid-catalysed hydrolysis of glycosides. J. Chem. Soc. 3429-3440 (1962).

66. Ranade, S. C. \& Demchenko, A. V. Mechanism of chemical glycosylation: focus on the mode of activation and departure of anomeric leaving groups. J. Carbohydr. Chem. 32, 1-43 (2013).

67. Castelli, R. et al. Activation of glycosyl halides by halogen bonding. Chem. Asian J. 9, 2095-2098 (2014)
68. Kobayashi, Y., Nakatsuji, Y., Li, S., Tsuzuki, S. \& Takemoto, Y. Direct Nglycofunctionalization of amides with glycosyl trichloroacetimidate by thiourea/halogen bond donor co-catalysis. Angew. Chem. Int. Ed. 57, 3646-3650 (2018).

69. Li, S., Kobayashi, Y. \& Takemoto, Y. Organocatalytic direct $\alpha$-selective $N$ glycosylation of amide with glycosyl trichloroacetimiate. Chem. Pharm. Bull. 66, 768-770 (2018).

70. Mumcu, A. \& Küçükbay, H. Determination of pKa values of some novel benzimidazole salts by using a new approach with ${ }^{1} \mathrm{H}$ NMR spectroscopy. Magn. Reson. Chem. 53, 1024-1030 (2015).

71. Dunn, M. H., Konstandaras, N., Cole, M. L. \& Harper, J. B. Targeted and systematic approach to the study of pKa values of imidazolium salts in dimethyl sulfoxide. J. Org. Chem. 82, 7324-7331 (2017).

72. Bell, R. P. The Tunnel Effect in Chemistry (University Press, Cambridge, 1980)

73. Kwart, $\mathrm{H}$. Temperature dependence of the primary kinetic isotopic effect as a mechanistic criterion. Acc. Chem. Res. 15, 401-408 (1982).

74. Schreiner, P. R. Tunneling control of chemical reactions: the third reactivity paradigm. J. Am. Chem. Soc. 139, 15276-15283 (2017).

75. Ley, D., Gerbig, D. \& Schreiner, P. R. Tunneling control of chemical reactions - the organic chemist's perspective. Org. Biomol. Chem. 10, 3781-3790 (2012).

76. Meisner, J. \& Kästner, J. Atom tunneling in chemistry. Angew. Chem. Int. Ed. 55, 5400-5413 (2016).

77. Nagel, Z. D. \& Klinman, J. P. Tunneling and dynamics in enzymatic hydride transfer. Chem. Rev. 106, 3095-3118 (2006).

78. More O' Ferrall, R. A. A pictorial representation of zero-point energy and tunneling contributions to the kinetic isotopic effect. J. Phys. Org. Chem. 23, 572-579 (2010).

79. Reyes, A. C., Amyes, T. L. \& Richard, J. P. Primary deuterium kinetic isotope effects: a probe for the origin of the rate acceleration for hydride transfer catalyzed by glyerol-3-phosphate dehydrogenase. Biochemistry 57, 4338-4348 (2018).

80. Truhlar, D. G. et al. The incorporation of quantum effects in enyzme kinetics modeling. Acc. Chem. Res. 35, 341-349 (2002).

81. Caldin, E. F. \& Mateo, S. Kinetic isotope effects in various solvents for the proton-transfer reactions of 4-nitrophenylnitromethane with bases. J. Chem. Soc. Chem. Commun. Issue 22, 854-855 (1973).

82. Caldin, E. F. \& Mateo, S. Kinetic isotope effects and tunnelling in the protontransfer reaction between 4-nitrophenylnitromethane and tetramethylguanidine in various aprotic solvents. J. Chem. Soc. Faraday Trans. 1 71, 1876-1904 (1975).

83. Mower, M. P. \& Blackmond, D. G. Mechanistic rationalization of unusual sigmoidal kinetic profiles in the Machetti-De Sarlo cycloaddition reaction. J. Am. Chem. Soc. 137, 2386-2391 (2015).

84. Sharma, K. et al. Cation-controlled enantio-and diastereoselective synthesis of indolines: an autoinductive phase-transfer initiated 5-endo-trig process. J. Am. Chem. Soc. 137, 13414-13424 (2015).

85. Keske, E. C., West, T. H. \& Lloyd-Jones, G. C. Analysis of autoinduction, inhibition and auto-inhibition in a Rh-catalyzed C-C cleavage: mechanism of decyanative aryl-silylation. ACS Catal. 8, 8932-8940 (2018).

86. Blackmond, D. An examination of the role of autocatalytic cycles in the chemistry of proposed primordial reactions. Angew. Chem. Int. Ed. 48, 386-390 (2009).

87. Bissette, A. J. \& Fletcher, S. P. Mechanisms of autocatalysis. Angew. Chem. Int Ed. 52, 12800-12826 (2013).

88. Plasson, R., Brandenburg, A., Jullien, L. \& Bersini, H. Autocatalyses. J. Phys. Chem. A 115, 8073-8085 (2011).

89. Kononov, L. O. Chemical reactivity and solution structure: on the way to a paradigm shift? RSC Adv. 5, 46718-46734 (2015).

90. Kononov, L. O. et al. Concentration dependence on glycosylation outcomes: a clue to reproducibility and understanding the reasons behind. Eur. J. Org. Chem. 2012, 1926-1934 (2012).

91. Yamamoto, H. \& Futatsugi, K. "Designer acids": combined acid catalysis for asymmetric synthesis. Angew. Chem. Int. Ed. 44, 1924-1942 (2005).

92. Peng, P. \& Schmidt, R. R. An alternative reaction course in $O$-glycosidation with $\mathrm{O}$-glycosyl trichloroacetimidates as glycosyl donors and Lewis acidic metal salts as catalyst: acid-base catalysis with gold chloride-glycosyl acceptor adducts. J. Am. Chem. Soc. 137, 12653-12659 (2015).

93. Kumar, A., Kumar, V., Dere, R. T. \& Schmidt, R. R. Glycoside bond formation via acid-base catalysis. Org. Lett. 13, 3612-3615 (2011).

94. Whitesides, G. M. \& Ismagilov, R. F. Complexity in chemistry. Science 284, 89-92 (1999).

95. Schreiner, P. R. \& Wittkopp, A. H-bonding additives act like Lewis acid catalysts. Org. Lett. 4, 217-220 (2002).

96. Kotke, M. \& Schreiner, P. R. Generally applicable organocatalytic tetrahydropyranylation of hydroxy functionalities with very low catalyst loading. Synthesis Issue 5, 779-790 (2007). 


\section{Acknowledgements}

We are grateful to Fonds der Chemischen Industrie for generous funding to C.C.J.L. through a Liebig fellowship. Prof. Herbert Waldmann is greatly acknowledged for his generous assistance and infrastructural support. C.X. acknowledges the Max Planck Society for postdoctoral funding and the Alexander von Humboldt Foundation for a postdoctoral fellowship. V.U.B.R. acknowledges the Max Planck Society for postdoctoral funding. We are grateful for infrastructural support from the Technical University of Dortmund and the Max Planck Institut für Molekulare Physiologie, Dortmund. Robert Lott is acknowledged for initial preliminary contributions to the project. Dr. Raphael Gasper-Schoenenbruecher of the Central Unit for Crystallography and Biophysics of the Max Planck Institut für Molekulare Physiologie is gratefully acknowledged for the ITC expertise and assistance. Jens Warmer is gratefully acknowledged for MALDI-TOF mass spectrometry assistance. Bernhard Griewel is gratefully acknowledged for NMR expertise and generous assistance in the in situ NMR monitoring experiments. The NMR department of the faculty of Chemistry and Chemical Biology of the Technical University of Dortmund is gratefully acknowledged for providing NMR timeslots for the NMR monitoring experiments.

\section{Author contributions}

C.C.J.L. conceived the idea and supervised the project. C.X. and V.U.B.R. conducted the majority of the experimental work. C.C.J.L, C.X., and V.U.B.R. prepared this manuscript. C.C.J.L., C.X., V.U.B.R., and J.W. contributed to data analysis and commented on the manuscript.

\section{Funding}

Open Access funding enabled and organized by Projekt DEAL.

\section{Competing interests}

The authors declare no competing interests.

\section{Additional information}

Supplementary information is available for this paper at https://doi.org/10.1038/s41467020-18595-2.

Correspondence and requests for materials should be addressed to C.C.J.L.

Peer review information Nature Communications thanks the anonymous reviewer(s) for their contribution to the peer review of this work.

Reprints and permission information is available at http://www.nature.com/reprints

Publisher's note Springer Nature remains neutral with regard to jurisdictional claims in published maps and institutional affiliations.

(c) (i) Open Access This article is licensed under a Creative Commons Attribution 4.0 International License, which permits use, sharing, adaptation, distribution and reproduction in any medium or format, as long as you give appropriate credit to the original author(s) and the source, provide a link to the Creative Commons license, and indicate if changes were made. The images or other third party material in this article are included in the article's Creative Commons license, unless indicated otherwise in a credit line to the material. If material is not included in the article's Creative Commons license and your intended use is not permitted by statutory regulation or exceeds the permitted use, you will need to obtain permission directly from the copyright holder. To view a copy of this license, visit http://creativecommons.org/ licenses/by/4.0/.

(C) The Author(s) 2020 